\title{
Synthesis of $\mathrm{BF}_{2}$ complex of 3-methylthio enaminones
}

\author{
Ti Zhang, ${ }^{\text {a,b }}$ Yue-Mei Jia, ${ }^{a}$ Sheng-Jiao Yan, ${ }^{a}$ Chu-Yi Yu, ${ }^{\text {a* }}$ and Zhi-Tang Huang ${ }^{\text {a* }}$ \\ ${ }^{a}$ Beijing National Laboratory for Molecular Sciences, CAS Key Laboratory of Molecular \\ Recognition and Function, Institute of Chemistry, Chinese Academy of Sciences, Beijing 100190, \\ People's Republic of China \\ ${ }^{b}$ Graduate University of the Chinese Academy of Sciences, Beijing 100049, People's Republic of \\ China \\ E-mail:yucy@iccas.ac.cn
}

\begin{abstract}
Novel $\mathrm{BF}_{2}$ complex of 3-methylthio enaminones or 3-methylthio enaminoketonatoboron difluorides have been synthesized via reactions of 3-methylthio enaminones with $\mathrm{BF}_{3} \cdot \mathrm{Et}_{2} \mathrm{O}$ in the presence of $\mathrm{Et}_{3} \mathrm{~N}$ in good to excellent yields.
\end{abstract}

Keywords: 3-Methylthio enaminones, ketene $N, S$-acetals, $\mathrm{BF}_{2}$ complexes, synthesis.

\section{Introduction}

Enaminones are useful synthetic intermediates that combine the ambident nucleophilicity of enamines with the ambident electrophilicity of enones, and their chemistry has received considerable attention continuously since their first appearance. ${ }^{1}$ Enaminones have proved to be versatile building blocks in organic synthesis, ${ }^{2}$ especially for the preparation of various heterocycles as potential agrochemicals or intermediates in dye and pharmaceutical industries. ${ }^{3}$ While their early use has been limited to serving as synthetic intermediates in organic synthesis, recent exploration on enaminones' applications in pharmaceutical development has made impressive progress. ${ }^{4}$ The therapeutic potential of these compounds has been realized in a number of areas, such as candidate agents as potential anticonvulsants and as potential modulators of multidrug resistance (MDR). ${ }^{4 a} 3$-Methylthio enaminones or ketene $N, S$-acetals, ${ }^{5}$ a special class of enaminones with a 3-methylthio substitution at the $\beta$-position, have attracted our attention due to the polyfunctional feature of these molecules. Because the MeS group is a good leaving group and could be subjected to a number of chemical transformations, the exploration on the synthetic utilities of 3-methylthio enaminones might result in the synthesis of a variety of novel nitrogen-containing heterocycles. In order to explore the chemical reactivity of 3methylthio enaminones, especially the region-selectivity of these bis-nucleophiles, we decided to 
make the $\mathrm{BF}_{2}$ complex of 3-methylthio enaminones and examine their chemical reactivity. While the synthesis of $\mathrm{BF}_{2}$ complexes of enaminones and their applications in organic synthesis have been reported before, ${ }^{6}$ the synthesis and applications of the $\mathrm{BF}_{2}$ complexes of 3-methylthio enaminones are yet to be explored. Due to the availability of a large number of enaminones, this might be resulted in the build-up of novel heterocyclic libraries of $\mathrm{BF}_{2}$ complexes which could be valuable entities for pharmaceutical and agrochemical applications.

\section{Results and Discussion}

3-Methylthio enaminones $\mathbf{3}$ have been synthesized via the reactions of ketene $S, S$-acetals $\mathbf{1}$ with various aryl substituents and the corresponding amines $\mathbf{2}$ according to the reported methods. ${ }^{8-13}$ The amino group and the carbonyl group of compound $\mathbf{3}$ are in Z-configuration due to the strong intramolecular hydrogen bonding between these two groups. Treatment of $\mathbf{3}$ with excess amount of $\mathrm{BF}_{3} \cdot \mathrm{Et}_{2} \mathrm{O}$ ( 5 equiv) in the presence of $\mathrm{Et}_{3} \mathrm{~N}$ followed by crystallization, afforded $\mathrm{BF}_{2}$ complex 4 in good to excellent yields (74-98\%). The reaction was conducted in the presence of $\mathrm{Et}_{3} \mathrm{~N}$ at room temperature for about $30 \mathrm{~min}$ (Scheme 1). The results are summarized in Table 1.

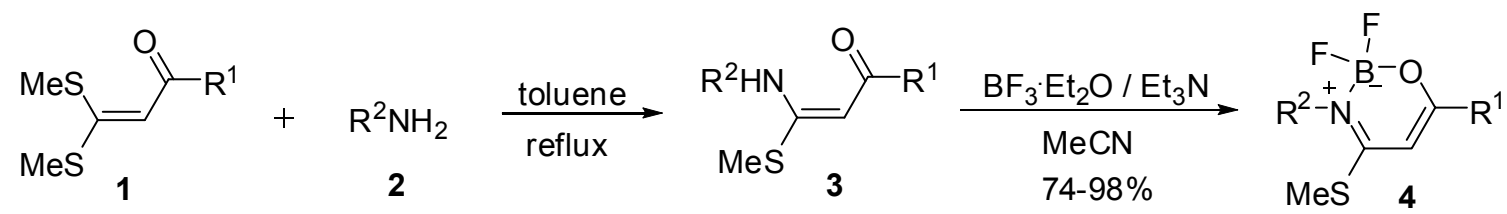

Scheme 1. Synthesis of $\mathrm{BF}_{2}$ complex from 3-methylthio enaminone.

As shown in Table 1, the reaction is barely affected by electronic and steric factors of the substitutions. All 3-methylthio enaminones, either with electron-donating or electronwithdrawing aryl groups underwent the reaction smoothly affording the desired products in high yields. Cyclohexyl substituent slightly decreased the yield of the reaction probably due to the steric effect (Table 1, entries 17, 18, 19). In the case of enaminone $\mathbf{3 e}$ which bears a fully substituted aryl group, the reaction had to be performed at $50^{\circ} \mathrm{CC}$ for $8 \mathrm{~h}$. in order to complete the reaction. Steric hindrance might be the cause of this observation (Table 1, entry 5).

All $\mathrm{BF}_{2}$ complexes were fully characterized by their spectral data. To take compound $\mathbf{4 f}$ as an example, in its IR spectrum, there was no signal which could be assigned to $\mathrm{C}=\mathrm{O}$ group; its ${ }^{1} \mathrm{H}-\mathrm{NMR}$ spectrum did not display the characteristic peak of $\mathrm{NH}$ group; and its ${ }^{13} \mathrm{C} \mathrm{NMR}$ spectrum displayed two characteristic signals at $\delta 174.0$ and 166.8 for the two tertiary carbon of $\mathrm{BF}_{2}$ complex at $\mathrm{C}-3$ and $\mathrm{C}-1$. These evidences, together with the mass spectrum and element analysis, indicated that the transformation of the enaminone structure to the iminium-enol structure and the involvement of the $\mathrm{N}, \mathrm{O}$-atoms of the 3-methylthio enaminones in the formation 
of the six-member ring $\mathrm{BF}_{2}$ complex. The structure of $\mathrm{BF}_{2}$ complex $\mathbf{4 f}$ was further proved by the single crystal X-ray analysis (Figure 1$)^{7}$

Table 1. Synthesis of $\mathrm{BF}_{2}$ complex $4 \mathbf{a}-\mathbf{s}$

\begin{tabular}{|c|c|c|c|c|c|c|c|}
\hline Entry & Reactant & $\mathrm{R}^{1}$ & $\mathrm{R}^{2}$ & Product & $\begin{array}{l}\text { Time } \\
(\mathrm{h})^{\mathrm{a}}\end{array}$ & $\begin{array}{l}\text { Yield } \\
(\%)^{b}\end{array}$ & $\begin{array}{l}\text { m.p. } \\
\left({ }^{\circ} \mathrm{C}\right)\end{array}$ \\
\hline 1 & $\mathbf{3 a}$ & $\mathrm{Ph}$ & $n-\operatorname{Pr}$ & $4 a$ & 0.5 & 88 & $134-135$ \\
\hline 2 & $\mathbf{3 b}$ & $p-\mathrm{CH}_{3}-\mathrm{Ph}$ & $n$-Pr & $4 b$ & 0.5 & 95 & $126-127$ \\
\hline 3 & $3 c$ & $p-\mathrm{Cl}-\mathrm{Ph}$ & $n-\operatorname{Pr}$ & $4 c$ & 0.5 & 98 & $138-139$ \\
\hline 4 & 3d & $p-\mathrm{CH}_{3} \mathrm{O}-\mathrm{Ph}$ & $n$-Pr & $4 d$ & 0.5 & 87 & $132-133$ \\
\hline 5 & $3 e$ & $\begin{array}{l}\text { 4-t-butyl-2,6- } \\
\text { dimethyl- } \\
\text { 3,5- } \\
\text { dinitrophenyl }\end{array}$ & $n-\operatorname{Pr}$ & $4 e$ & $8^{c}$ & 89 & $146-147$ \\
\hline 6 & $3 f$ & $p$-phenyl-Ph & $n-\operatorname{Pr}$ & $4 f$ & 0.5 & 90 & $141-142$ \\
\hline 7 & $3 g$ & $p$-F-Ph & $n-\operatorname{Pr}$ & $4 g$ & 0.5 & 92 & $138-139$ \\
\hline 8 & $3 \mathbf{h}$ & $p-\mathrm{CF}_{3}-\mathrm{Ph}$ & $n-\operatorname{Pr}$ & $4 h$ & 0.5 & 88 & $105-106$ \\
\hline 9 & $3 \mathbf{i}$ & $\mathrm{Ph}$ & $\mathrm{Ph}$ & $4 i$ & 0.5 & 91 & 192-194 \\
\hline 10 & $\mathbf{3 j}$ & $\mathrm{Ph}$ & $i-\operatorname{Pr}$ & $4 j$ & 0.5 & 96 & $132-134$ \\
\hline 11 & $3 \mathbf{k}$ & $\mathrm{Ph}$ & $n-\mathrm{Bu}$ & $4 k$ & 0.5 & 90 & $101-102$ \\
\hline 12 & 31 & $\mathrm{Ph}$ & $\mathrm{CH}_{2} \mathrm{CH}=\mathrm{CH}_{2}$ & 41 & 0.5 & 86 & $135-137$ \\
\hline 13 & $3 \mathrm{~m}$ & $\mathrm{Ph}$ & cyclohexyl & $4 m$ & 0.5 & 93 & $144-145$ \\
\hline 14 & $3 n$ & $p-\mathrm{CH}_{3}-\mathrm{Ph}$ & $\mathrm{Ph}$ & $4 n$ & 0.5 & 90 & $202-203$ \\
\hline 15 & 30 & $p$-Cl-Ph & $\mathrm{Ph}$ & 40 & 0.5 & 91 & 189-190 \\
\hline 16 & $3 p$ & $p$-F-Ph & $\mathrm{Ph}$ & $4 p$ & 0.5 & 93 & $178-179$ \\
\hline 17 & $3 q$ & $p-\mathrm{CH}_{3}-\mathrm{Ph}$ & cyclohexyl & $4 q$ & 0.5 & 85 & $155-156$ \\
\hline 18 & $3 r$ & $p$-Cl-Ph & cyclohexyl & $4 r$ & 0.5 & 83 & $173-174$ \\
\hline 19 & $3 s$ & $p-\mathrm{CH}_{3} \mathrm{O}-\mathrm{Ph}$ & cyclohexyl & $4 s$ & 0.5 & 74 & 137-139 \\
\hline
\end{tabular}

${ }^{a}$ Monitored by TLC analysis. ${ }^{b}$ Isolated yields by crystallization of the crude product from petroleum ether and ethyl acetate at room temperature. ${ }^{\mathrm{c}}$ The reaction was performed at $50^{\circ} \mathrm{C}$ for $8 \mathrm{~h}$.

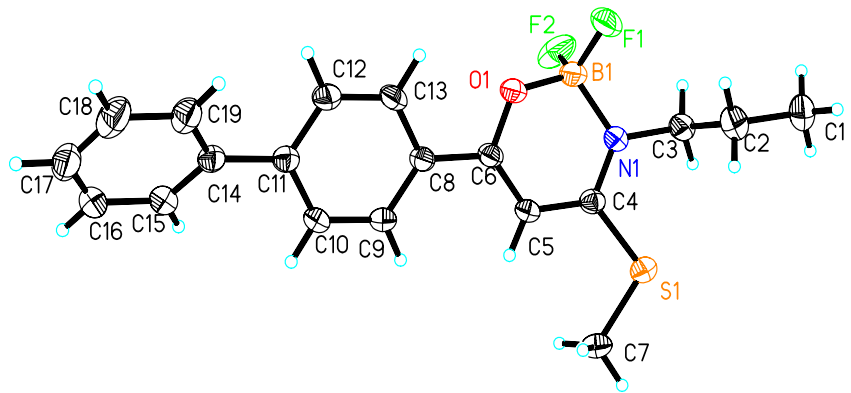

Figure 1. X-ray structure of $\mathbf{4 f}$. 


\section{Conclusions}

In summary, novel $\mathrm{BF}_{2}$ complex of 3-methylthio enaminones have been synthesized efficiently via the reactions of 3-methylthio enaminones with $\mathrm{BF}_{3} \cdot \mathrm{Et}_{2} \mathrm{O}$ in the presence of $\mathrm{Et}_{3} \mathrm{~N}$ in good to excellent yields. These novel compounds will be helpful for us to better understand the chemical reactivity of enaminones and might be valuable in pharmaceutical and agrochemical applications.

\section{Experimental Section}

General. Melting points were determined using an electrothermal melting point apparatus and were uncorrected. IR spectra were recorded on a JASCO FT/IR-480 spectrometer using $\mathrm{KBr}$ pellets. ${ }^{1} \mathrm{H}$ NMR $(300 \mathrm{MHz})$ and ${ }^{13} \mathrm{C}$ NMR $(75 \mathrm{MHz})$ were recorded on a Bruker AV300 magnetic resonance spectrometer in $\mathrm{CDCl}_{3}$ solution. Chemical shifts $(\delta)$ are reported in ppm with tetramethylsilane as internal standard. High resolution mass spectra (HRMS) were recorded on an APEXII FT-ICR spectrometer with EI mode. Elemental analysis was performed at the Center for Instrumental Analysis of ICCAS.

\section{General procedure for the synthesis of 3-methylthio enaminone}

A solution of ketene $S, S$-acetals 1 (1.0 equiv) and corresponding amines 2 (2.0-5.0 equiv) in toluence $(30 \mathrm{~mL})$ was heated to reflux. When TLC indicated the completion of the reaction, the resulting reaction mixture was concentrated in vacuo. The residue was purified by column chromatography (silica gel, AcOEt-petroleum ether: $\mathrm{v} / \mathrm{v}=1 / 15-1 / 8$ ) affording the desired ketene $N, S$-acetals 3 .

(E)-3-(Methylthio)-1-phenyl-3-(propylamino)prop-2-en-1-one (3a). The known compound 3a was made according to the reported method. ${ }^{8}$ Reaction of $S, S$-acetal $1\left(\mathrm{R}^{1}=\mathrm{Ph}, 1.12 \mathrm{~g}, 5.0\right.$ $\mathrm{mmol})$ and $n$-propylamine $(25.0 \mathrm{mmol})$ generated the product $3 \mathbf{a}(965 \mathrm{mg}, 82 \%)$ as a yellow oil. FT-IR (film/ $\mathrm{cm}^{-1}$ ): 3059w, 2963m, 2930m, 2873m, 1560s, 1496s, 1471s, 1436s, 1273s, 721s, 641s; ${ }^{1} \mathrm{H}$ NMR (300 MHz, $\left.\mathrm{CDCl}_{3}\right): \delta 11.92(\mathrm{~s}, 1 \mathrm{H}, \mathrm{NH}), 7.87-7.83(\mathrm{~m}, 2 \mathrm{H}, \mathrm{PhH}), 7.38-7.33(\mathrm{~m}$, $3 \mathrm{H}, \mathrm{PhH}), 5.61(\mathrm{~s}, 1 \mathrm{H},=\mathrm{CH}), 3.31-2.25\left(\mathrm{~m}, 2 \mathrm{H}, \mathrm{NHCH}_{2}\right), 2.34\left(\mathrm{~s}, 3 \mathrm{H}, \mathrm{SCH}_{3}\right), 1.69-1.62(\mathrm{~m}, 2 \mathrm{H}$, $\left.\mathrm{CH}_{2}\right), 1.01\left(\mathrm{t}, J=7.4 \mathrm{~Hz}, 3 \mathrm{H}, \mathrm{CH}_{3}\right) \mathrm{ppm} ;{ }^{13} \mathrm{C} \mathrm{NMR}\left(75 \mathrm{MHz}, \mathrm{CDCl}_{3}\right): \delta 184.8,169.6,140.7$, 130.3, 128.1, 126.8, 86.0, 45.6, 22.8, 14.1, $11.4 \mathrm{ppm}$.

(E)-3-(Methylthio)-3-(propylamino)-1-p-tolylprop-2-en-1-one (3b). The known compound 3b was made according to the reported method. ${ }^{9}$ Reaction of $S, S$-acetal $\mathbf{1}\left(\mathrm{R}^{1}=p\right.$ - $\mathrm{CH}_{3}-\mathrm{Ph}, 2.38 \mathrm{~g}$, $10.0 \mathrm{mmol})$ and $n$-propylamine $(50.0 \mathrm{mmol})$ generated the product $\mathbf{3 b}(1.94 \mathrm{~g}, 78 \%)$ as a yellow solid. m.p. $36-38^{\circ} \mathrm{C}$ (EtOAc); FT-IR (film/ $\mathrm{cm}^{-1}$ ): 3024w, 2963m, 2928m, 2873m, 1566s, 1472s, 1272s, 1078m, 767s, 705m; ${ }^{1} \mathrm{H}$ NMR (300 MHz, $\mathrm{CDCl}_{3}$ ): $\delta 11.88$ (s, 1H, NH), 7.76 (d, $J=7.1$ $\mathrm{Hz}, 2 \mathrm{H}, \mathrm{PhH}), 7.17(\mathrm{~d}, J=6.7 \mathrm{~Hz}, 2 \mathrm{H}, \mathrm{PhH}), 5.61(\mathrm{~s}, 1 \mathrm{H},=\mathrm{CH}), 3.28-3.22\left(\mathrm{~m}, 2 \mathrm{H}, \mathrm{NHC}_{2}\right)$, 2.36-2.33 (m, 6H, $\left.\mathrm{SCH}_{3}, \mathrm{PhCH}_{3}\right), 1.70-1.65\left(\mathrm{~m}, 2 \mathrm{H}, \mathrm{CH}_{2}\right), 1.02\left(\mathrm{t}, J=7.2 \mathrm{~Hz}, 3 \mathrm{H}, \mathrm{CH}_{3}\right) \mathrm{ppm}$; ${ }^{13} \mathrm{C}$ NMR $\left(75 \mathrm{MHz}, \mathrm{CDCl}_{3}\right): \delta 184.8,169.3,140.6,138.0,128.9,126.9,85.8,45.7,22.9,21.3$, 
14.1, 11.5 ppm; LRMS (ESI, positive) $\mathrm{m} / z$ 250.2 [M+H] $]^{+}$; Anal.calcd for $\mathrm{C}_{14} \mathrm{H}_{19} \mathrm{NOS}$ : C, 67.43; H, 7.68; N, 5.62. Found: C, 67.14; H, 7.68; N, 5.66.

(E)-1-(4-Chlorophenyl)-3-(methylthio)-3-(propylamino)prop-2-en-1-one (3c). The known compound $3 \mathbf{c}$ was made according to the reported method. ${ }^{10}$ Reaction of $S, S$-acetal $\mathbf{1}\left(\mathrm{R}^{1}=p\right.$-Cl$\mathrm{Ph}, 2.59 \mathrm{~g}, 10.0 \mathrm{mmol})$ and $n$-propylamine $(50.0 \mathrm{mmol})$ generated the product $3 \mathrm{c}(2.28 \mathrm{~g}, 84 \%)$ as a slightly yellow solid. m.p. $69-71^{\circ} \mathrm{C}$ (EtOAc); FT-IR (film/ $\mathrm{cm}^{-1}$ ): $2963 \mathrm{~m}, 2930 \mathrm{~m}, 2873 \mathrm{w}$, 1559s, 1471s, 1272s, 1091s, 1011m, 846m, 763s; ${ }^{1} \mathrm{H}$ NMR (300 MHz, $\left.\mathrm{CDCl}_{3}\right): \delta 11.88(\mathrm{~s}, 1 \mathrm{H}$, $\mathrm{NH}), 7.80(\mathrm{~d}, J=8.5 \mathrm{~Hz}, 2 \mathrm{H}, \mathrm{PhH}), 7.38(\mathrm{~d}, J=8.5 \mathrm{~Hz}, 2 \mathrm{H}, \mathrm{PhH}), 5.59$ (s, 1H, =CH), 3.39-3.32 $\left(\mathrm{m}, 2 \mathrm{H}, \mathrm{NHC}_{2}\right), 2.47\left(\mathrm{~s}, 3 \mathrm{H}, \mathrm{SCH}_{3}\right), 1.76-1.64\left(\mathrm{~m}, 2 \mathrm{H}, \mathrm{CH}_{2}\right), 1.06$ (t, $\left.J=7.4 \mathrm{~Hz}, 3 \mathrm{H}, \mathrm{CH}_{3}\right)$ ppm; ${ }^{13} \mathrm{C}$ NMR (75 MHz, $\left.\mathrm{CDCl}_{3}\right): \delta 183.6,170.0,139.2,136.4,128.4,128.3,85.8,45.8,22.8$, 14.3, 11.5 ppm; LRMS (ESI, positive) $\mathrm{m} / z$ 270.1 $[\mathrm{M}+\mathrm{H}]^{+}$; Anal.calcd for $\mathrm{C}_{13} \mathrm{H}_{16} \mathrm{ClNOS}$ : C, 57.87; H, 5.98; N, 5.19. Found: C, 57.69; H, 5.94; N, 5.20.

(E)-1-(4-Methoxyphenyl)-3-(methylthio)-3-(propylamino)prop-2-en-1-one (3d). The known compound 3d was made according to the reported method. ${ }^{9}$ Reaction of $S, S$-acetal $\mathbf{1}\left(\mathrm{R}^{1}=p\right.$ $\left.\mathrm{CH}_{3} \mathrm{O}-\mathrm{Ph}, 717 \mathrm{mg}, 2.8 \mathrm{mmol}\right)$ and $n$-propylamine $(14.0 \mathrm{mmol})$ generated the product $3 \mathrm{~d}(636 \mathrm{mg}$, $85 \%$ ) as a slightly yellow solid. m.p. $42-44^{\circ} \mathrm{C}$ (EtOAc); FT-IR (film/ $\left./ \mathrm{cm}^{-1}\right): 2962 \mathrm{~m}, 2931 \mathrm{~m}$, 2873w, 1565s, 1472s, 1251s, 1169s, $1031 \mathrm{~m}, 771 \mathrm{~m} ;{ }^{1} \mathrm{H}$ NMR $\left(300 \mathrm{MHz}, \mathrm{CDCl}_{3}\right): \delta 11.82(\mathrm{~s}, 1 \mathrm{H}$, $\mathrm{NH}), 7.84(\mathrm{~d}, J=8.8 \mathrm{~Hz}, 2 \mathrm{H}, \mathrm{PhH}), 6.88(\mathrm{~d}, J=8.8 \mathrm{~Hz}, 2 \mathrm{H}, \mathrm{PhH}), 5.59(\mathrm{~s}, 1 \mathrm{H},=\mathrm{CH}), 3.76-3.70$ $\left(\mathrm{s}, 3 \mathrm{H}, \mathrm{OCH}_{3}\right), 3.33-3.26\left(\mathrm{~m}, 2 \mathrm{H}, \mathrm{NHCH}_{2}\right), 2.38\left(\mathrm{~s}, 3 \mathrm{H}, \mathrm{SCH}_{3}\right), 1.71-1.63\left(\mathrm{~m}, 2 \mathrm{H}, \mathrm{CH}_{2}\right), 1.02(\mathrm{t}$, $\left.J=7.4 \mathrm{~Hz}, 3 \mathrm{H}, \mathrm{CH}_{3}\right) \mathrm{ppm} ;{ }^{13} \mathrm{C} \mathrm{NMR}\left(75 \mathrm{MHz}, \mathrm{CDCl}_{3}\right): \delta 184.2,168.9,161.5,133.2,128.6$, 113.3, 85.5, 55.2, 45.6, 22.9, 14.1, 11.4 ppm; LRMS (ESI, positive) $\mathrm{m} / z 266.2[\mathrm{M}+\mathrm{H}]^{+}$; Anal.calcd for $\mathrm{C}_{14} \mathrm{H}_{19} \mathrm{NO}_{2} \mathrm{~S}$ : C, 63.36; H, 7.22; N, 5.28. Found: C, 63.21; H, 7.19; N, 5.38.

(E)-1-(4-tert-Butyl-2,6-dimethyl-3,5-dinitrophenyl)-3-(methylthio)-3-(propylamino)

prop-2-en-1-one (3e). Using the general procedure, reaction of $S, S$-acetal $1\left(\mathrm{R}^{1}=4\right.$ - $t$-butyl-2,6dimethyl-3,5-dinitrophenyl, $2.04 \mathrm{~g}, 5.0 \mathrm{mmol})$ and $n$-propylamine $(25.0 \mathrm{mmol})$ generated the product 3e (1.85 g, 89\%) as a white solid. m.p. 42-44 ${ }^{\circ} \mathrm{C}\left(\right.$ EtOAc); FT-IR (film/cm $\left.{ }^{-1}\right): 2967 \mathrm{~m}$, 2931m, 2875w, 1539s, 1474s, 1352s, 1275s; ${ }^{1} \mathrm{H}$ NMR (300 MHz, $\left.\mathrm{CDCl}_{3}\right): \delta 11.62(\mathrm{~s}, 1 \mathrm{H}, \mathrm{NH})$, $4.98(\mathrm{~s}, 1 \mathrm{H},=\mathrm{CH}), 3.41-3.34\left(\mathrm{~m}, 2 \mathrm{H}, \mathrm{NHCH}_{2}\right), 2.36\left(\mathrm{~s}, 3 \mathrm{H}, \mathrm{SCH}_{3}\right), 2.17\left(\mathrm{~s}, 6 \mathrm{H}, 2 \mathrm{PhCH}_{3}\right), 1.79-$ $1.71\left(\mathrm{~m}, 2 \mathrm{H}, \mathrm{CH}_{2}\right), 1.44\left(\mathrm{~s}, 9 \mathrm{H}, 3 \mathrm{CH}_{3}\right), 1.06\left(\mathrm{t}, J=7.4 \mathrm{~Hz}, 3 \mathrm{H}, \mathrm{CH}_{3}\right) \mathrm{ppm} ;{ }^{13} \mathrm{C} \mathrm{NMR}(75 \mathrm{MHz}$, $\left.\mathrm{CDCl}_{3}\right): \delta 185.3,171.3,150.2,144.3,130.2,128.2,89.8,46.0,37.1,30.3,22.6,15.2,14.2,11.3$ ppm; LRMS (ESI, positive) $\mathrm{m} / z 410.4[\mathrm{M}+\mathrm{H}]^{+}$; Anal.calcd for $\mathrm{C}_{19} \mathrm{H}_{27} \mathrm{~N}_{3} \mathrm{O}_{5} \mathrm{~S}$ : C, 55.73; H, 6.65; N, 10.26. Found: C, 55.62; H, 6.74; N, 9.91.

(E)-3-(Methylthio)-1-(4-phenylphenyl)-3-(propylamino)prop-2-en-1-one (3f). Using the general procedure, reaction of $S, S$-acetal $1(1.54 \mathrm{~g}, 5.1 \mathrm{mmol})$ and $n$-propylamine $\left(\mathrm{R}^{1}=p\right.$ phenyl-Ph, $25.5 \mathrm{mmol})$ generated the product $3 \mathrm{f}(1.15 \mathrm{~g}, 72 \%)$ as a yellow oil. FT-IR (film $\left./ \mathrm{cm}^{-1}\right)$ : 3029w, 2962w, 2929w, 2872w, 1559s, 1471s, 1276s, 749s, 698m; $\left.{ }^{1} \mathrm{H} \mathrm{NMR} \mathrm{(300} \mathrm{MHz,} \mathrm{CDCl} 3\right)$ : $\delta 11.97(\mathrm{t}, J=4.8 \mathrm{~Hz}, 1 \mathrm{H}, \mathrm{NH}), 7.94-7.92(\mathrm{~m}, 2 \mathrm{H}, \mathrm{PhH}), 7.61-7.56(\mathrm{~m}, 4 \mathrm{H}, \mathrm{PhH}), 7.40-7.23(\mathrm{~m}$, $3 \mathrm{H}, \mathrm{PhH}), 5.65(\mathrm{~s}, 1 \mathrm{H},=\mathrm{CH}), 3.30-3.24\left(\mathrm{~m}, 2 \mathrm{H}, \mathrm{NHCH}_{2}\right), 2.33\left(\mathrm{~s}, 3 \mathrm{H}, \mathrm{SCH}_{3}\right), 1.69-1.62(\mathrm{~m}$, $\left.2 \mathrm{H}, \mathrm{CH}_{2}\right), 1.01$ (t, $\left.J=7.3 \mathrm{~Hz}, 3 \mathrm{H}, \mathrm{CH}_{3}\right) \mathrm{ppm} ;{ }^{13} \mathrm{C} \mathrm{NMR}\left(75 \mathrm{MHz}, \mathrm{CDCl}_{3}\right): \delta 184.4,169.6$, 
143.0, 140.4, 139.6, 128.9, 127.7, 127.5, 127.1, 126.9, 86.2, 45.8, 22.9, 14.2, 11.6 ppm; HRMS (EI) Calcd for $\mathrm{C}_{19} \mathrm{H}_{21} \mathrm{NOS}[\mathrm{M}]^{+} 311.1344$, Found 311.1347.

(E)-1-(4-Fluorophenyl)-3-(methylthio)-3-(propylamino)prop-2-en-1-one (3g). Using the general procedure, reaction of $S, S$-acetal $1\left(\mathrm{R}^{1}=p\right.$-F-Ph, $\left.1.33 \mathrm{~g}, 5.5 \mathrm{mmol}\right)$ and $n$-propylamine $(27.5 \mathrm{mmol})$ generated the product $3 \mathrm{~g}(1.27 \mathrm{~g}, 92 \%)$ as a yellow oil. FT-IR (film $\left./ \mathrm{cm}^{-1}\right): 2964 \mathrm{~m}$, $2931 \mathrm{~m}, 2874 \mathrm{w}, 1569 \mathrm{~s}, 1474 \mathrm{~s}, 1273 \mathrm{~s}, 1231 \mathrm{~s}, 1153 \mathrm{~s}, 849 \mathrm{~m}, 768 \mathrm{~s}, 595 \mathrm{~s} ;{ }^{1} \mathrm{H}$ NMR $(300 \mathrm{MHz}$, $\left.\mathrm{CDCl}_{3}\right): \delta 11.87(\mathrm{~s}, 1 \mathrm{H}, \mathrm{NH}), 7.88-7.83(\mathrm{~m}, 2 \mathrm{H}, \mathrm{PhH}), 7.06-7.00(\mathrm{~m}, 2 \mathrm{H}, \mathrm{PhH}), 5.56(\mathrm{~s}, 1 \mathrm{H}$, $=\mathrm{CH}), 3.32-3.25\left(\mathrm{~m}, 2 \mathrm{H}, \mathrm{NHCH}_{2}\right), 2.37\left(\mathrm{~s}, 3 \mathrm{H}, \mathrm{SCH}_{3}\right), 1.70-1.63\left(\mathrm{~m}, 2 \mathrm{H}, \mathrm{CH}_{2}\right), 1.02(\mathrm{t}, J=7.4$ $\left.\mathrm{Hz}, 3 \mathrm{H}, \mathrm{CH}_{3}\right) \mathrm{ppm} ;{ }^{13} \mathrm{C} \mathrm{NMR}\left(75 \mathrm{MHz}, \mathrm{CDCl}_{3}\right): \delta 183.2,169.6,165.5\left(\mathrm{~d},{ }^{1} J_{\mathrm{C}-\mathrm{F}}=247.5 \mathrm{~Hz}, 1 \mathrm{C}\right.$, $\mathrm{C}-\mathrm{F}), 136.8\left(\mathrm{~d},{ }^{4} J_{\mathrm{C}-\mathrm{F}}=3.0 \mathrm{~Hz}, 1 \mathrm{C}\right), 129.0\left(\mathrm{~d},{ }^{3} J_{\mathrm{C}-\mathrm{F}}=8.5 \mathrm{~Hz}, 2 \mathrm{C}\right), 114.8\left(\mathrm{~d},{ }^{2} J_{\mathrm{C}-\mathrm{F}}=21.0 \mathrm{~Hz}\right.$, 2C), 85.5, 45.6, 22.7, 13.9, 11.3 ppm; HRMS (EI) Calcd for $\mathrm{C}_{13} \mathrm{H}_{16} \mathrm{FNOS}[\mathrm{M}]^{+}$253.0937, Found 253.0939 .

(E)-1-(4-(Trifluoromethyl)phenyl)-3-(methylthio)-3-(propylamino)prop-2-en-1-one $\quad(3 \mathrm{~h})$. Using the general procedure, reaction of $S, S$-acetal $1\left(\mathrm{R}^{1}=p-\mathrm{CF}_{3}-\mathrm{Ph}, 1.57 \mathrm{~g}, 5.4 \mathrm{mmol}\right)$ and $n$ propylamine $(25.0 \mathrm{mmol})$ generated the product $\mathbf{3 h}(1.45 \mathrm{~g}, 89 \%)$ as a yellow solid. m.p. 43$44^{\circ} \mathrm{C}$ (EtOAc); FT-IR (film/ $\mathrm{cm}^{-1}$ ): 2966w, 2933w, 2876w, 1566s, 1478s, 1324s, 1124s, 1015m; ${ }^{1} \mathrm{H}$ NMR (300 MHz, $\left.\mathrm{CDCl}_{3}\right): \delta 12.00(\mathrm{~s}, 1 \mathrm{H}, \mathrm{NH}), 7.96(\mathrm{~d}, J=8.1 \mathrm{~Hz}, 2 \mathrm{H}, \mathrm{PhH}), 7.64(\mathrm{~d}, J=8.1$ $\mathrm{Hz}, 2 \mathrm{H}, \mathrm{PhH}), 5.62(\mathrm{~s}, 1 \mathrm{H},=\mathrm{CH}), 3.35-3.28\left(\mathrm{~m}, 2 \mathrm{H}, \mathrm{NHCH}_{2}\right), 2.41\left(\mathrm{~s}, 3 \mathrm{H}, \mathrm{SCH}_{3}\right), 1.73-1.66(\mathrm{~m}$, $\left.2 \mathrm{H}, \mathrm{CH}_{2}\right), 1.04\left(\mathrm{t}, J=7.4 \mathrm{~Hz}, 3 \mathrm{H}, \mathrm{CH}_{3}\right) \mathrm{ppm} ;{ }^{13} \mathrm{C} \mathrm{NMR}\left(75 \mathrm{MHz}, \mathrm{CDCl}_{3}\right): \delta 182.8,170.5$, $144.0,131.6\left(\mathrm{q},{ }^{2} J_{\mathrm{C}-\mathrm{F}}=30.0 \mathrm{~Hz}, 1 \mathrm{C}, \underline{\mathrm{CCF}}_{3}\right), 124.0\left(\mathrm{q},{ }^{1} J_{\mathrm{C}-\mathrm{F}}=270.0 \mathrm{~Hz}, 1 \mathrm{C}, \underline{\mathrm{CF}}_{3}\right), 127.1,125.0$ $\left(\mathrm{q},{ }^{3} J_{\mathrm{C}-\mathrm{F}}=5.6 \mathrm{~Hz}, 2 \mathrm{C}\right), 86.0,45.7,22.6,13.9,11.2 \mathrm{ppm}$; LRMS (ESI, positive) $\mathrm{m} / \mathrm{z} 304.1$ $[\mathrm{M}+\mathrm{H}]^{+}$; Anal.calcd for $\mathrm{C}_{14} \mathrm{H}_{16} \mathrm{~F}_{3} \mathrm{NOS}$ : C, 55.43; H, 5.32; N, 4.62. Found: C, 55.53; H, 5.33; N, 4.83.

(E)-3-(Methylthio)-1-phenyl-3-(phenylamino)prop-2-en-1-one (3i). The known compound 3i was made according to the reported method. ${ }^{11}$ Reaction of $S, S$-acetal $\mathbf{1}\left(\mathrm{R}^{1}=\mathrm{Ph}, 1.12 \mathrm{~g}, 5.0\right.$ $\mathrm{mmol})$ and aniline $(15.0 \mathrm{mmol})$ generated the product $3 \mathbf{i}(942 \mathrm{mg}, 70 \%)$ as a yellow solid. m.p. $55-56^{\circ} \mathrm{C}$ (EtOAc); FT-IR (film/ $\left.\mathrm{cm}^{-1}\right): 3060 \mathrm{~m}, 2925 \mathrm{~s}, 1557 \mathrm{vs}, 1466 \mathrm{~s}, 1431 \mathrm{~s}, 1298 \mathrm{~s}, 1261 \mathrm{~s}, 729 \mathrm{~s}$, $692 \mathrm{~s} ;{ }^{1} \mathrm{H}$ NMR (300 MHz, $\left.\mathrm{CDCl}_{3}\right): \delta 13.58(\mathrm{~s}, 1 \mathrm{H}, \mathrm{NH}), 7.92-7.89(\mathrm{~m}, 2 \mathrm{H}, \mathrm{PhH}), 7.41-7.17(\mathrm{~m}$, $8 \mathrm{H}, \mathrm{PhH}), 5.86(\mathrm{~s}, 1 \mathrm{H},=\mathrm{CH}), 2.32\left(\mathrm{~s}, 3 \mathrm{H}, \mathrm{SCH}_{3}\right) \mathrm{ppm} ;{ }^{13} \mathrm{C} \mathrm{NMR}\left(75 \mathrm{MHz}, \mathrm{CDCl}_{3}\right): \delta 186.1$, 167.6, 140.3, 138.3, 131.0, 129.1, 128.4, 127.2, 126.4, 125.2, 88.9, 14.8 ppm; LRMS (ESI, positive) $\mathrm{m} / \mathrm{z} 270.2[\mathrm{M}+\mathrm{H}]^{+}$; Anal.calcd for $\mathrm{C}_{16} \mathrm{H}_{15} \mathrm{NOS}: \mathrm{C}, 71.34 ; \mathrm{H}, 5.61$; N, 5.20. Found: C, 71.17; H, 5.68; N, 5.34. $\quad\left[\mathrm{lit}^{4}\right.$ : m.p. $56-57^{\circ} \mathrm{C} ;{ }^{1} \mathrm{H}$ NMR: $\delta 8.0-8.3(\mathrm{~m}, 2 \mathrm{H}), 7.4-7.8(\mathrm{~m}, 8 \mathrm{H})$, $6.11(\mathrm{~s}, 1 \mathrm{H}, \mathrm{H}-2), 2.50\left(\mathrm{~s}, 3 \mathrm{H}, \mathrm{SCH}_{3}\right) \mathrm{ppm}$.]

(E)-3-(Isopropylamino)-3-(methylthio)-1-phenylprop-2-en-1-one (3j). The known compound 3j was made according to the reported method. ${ }^{8}$ Reaction of $S, S$-acetal $\mathbf{1}\left(\mathrm{R}^{1}=\mathrm{Ph}, 1.07 \mathrm{~g}, 4.8\right.$ $\mathrm{mmol}$ ) and isopropylamine (24.0 mmol) generated the product $3 \mathbf{j}(974 \mathrm{mg}, 87 \%)$ as a yellow oil. FT-IR (film/ $\left.\mathrm{cm}^{-1}\right)$ : 3058w, 2970m, 2927w, 2871w, 1565s, 1469s, 1279s, $1155 \mathrm{~m}, 722 \mathrm{~s}, 641 \mathrm{~m} ;{ }^{1} \mathrm{H}$ NMR (300 MHz, $\left.\mathrm{CDCl}_{3}\right): \delta 11.91(\mathrm{~d}, J=8.1 \mathrm{~Hz}, 1 \mathrm{H}, \mathrm{NH}), 7.87-7.84(\mathrm{~m}, 2 \mathrm{H}, \mathrm{PhH}), 7.37-7.33$ $(\mathrm{m}, 3 \mathrm{H}, \mathrm{PhH}), 5.59(\mathrm{~s}, 1 \mathrm{H},=\mathrm{CH}), 3.91-3.84(\mathrm{~m}, 1 \mathrm{H}, \mathrm{NHC} \underline{\mathrm{H}}), 2.34\left(\mathrm{~s}, 3 \mathrm{H}, \mathrm{SCH}_{3}\right), 1.27(\mathrm{t}, J=$ 
$6.3 \mathrm{~Hz}, 6 \mathrm{H}) \mathrm{ppm} ;{ }^{13} \mathrm{C} \mathrm{NMR}\left(75 \mathrm{MHz}, \mathrm{CDCl}_{3}\right): \delta 184.7,168.1,140.7,130.3,128.1,126.8,85.8$, 46.0, 23.2, $14.1 \mathrm{ppm}$. (lit ${ }^{8}$ : no data reported)

(E)-3-(Butylamino)-3-(methylthio)-1-phenylprop-2-en-1-one (3k). The known compound 3k was made according to the reported method. ${ }^{12}$ Reaction of $S, S$-acetal $1\left(\mathrm{R}^{1}=\mathrm{Ph}, 1.05 \mathrm{~g}, 4.7\right.$ $\mathrm{mmol})$ and $n$-butylamine $(23.5 \mathrm{mmol})$ generated the product $3 \mathbf{k}(906 \mathrm{mg}, 78 \%)$ as a yellow oil. FT-IR (film/ $\mathrm{cm}^{-1}$ ): 3058w, 2957s, 2929s, 2871m, 1559vs, 1471s, $1275 \mathrm{~s}, 1066 \mathrm{~m}, 721 \mathrm{~s}, 641 \mathrm{~m} ;{ }^{1} \mathrm{H}$ NMR (300 MHz, $\left.\mathrm{CDCl}_{3}\right): \delta 11.91(\mathrm{~s}, 1 \mathrm{H}, \mathrm{NH}), 7.87-7.84(\mathrm{~m}, 2 \mathrm{H}, \mathrm{PhH}), 7.36-7.34(\mathrm{~m}, 3 \mathrm{H}$, $\mathrm{PhH}), 5.61(\mathrm{~s}, 1 \mathrm{H},=\mathrm{CH}), 3.34-3.27\left(\mathrm{~m}, 2 \mathrm{H}, \mathrm{NHC}_{2}\right), 2.33\left(\mathrm{~s}, 3 \mathrm{H}, \mathrm{SCH}_{3}\right), 1.66-1.56(\mathrm{~m}, 2 \mathrm{H}$, $\left.\mathrm{CH}_{2}\right), 1.45-1.38\left(\mathrm{~m}, 2 \mathrm{H}, \mathrm{CH}_{2}\right), 0.95(\mathrm{t}, J=7.2 \mathrm{~Hz}, 3 \mathrm{H}) \mathrm{ppm} ;{ }^{13} \mathrm{C} \mathrm{NMR}\left(75 \mathrm{MHz}, \mathrm{CDCl}_{3}\right): \delta$ $184.7,169.5,140.7,130.3,128.1,126.8,85.9,43.6,31.5,20.0,14.1,13.7$ ppm. (lit ${ }^{12}$ : no data reported)

(E)-3-(Allylamino)-3-(methylthio)-1-phenylprop-2-en-1-one (3I). Using the general procedure, reaction of $S, S$-acetal $1\left(\mathrm{R}^{1}=\mathrm{Ph}, 1.16 \mathrm{~g}, 5.2 \mathrm{mmol}\right)$ and allylamine $(26.0 \mathrm{mmol})$ generated the product $3 \mathrm{l}(1.04 \mathrm{~g}, 86 \%)$ as a red oil. FT-IR (film/ $\left.\mathrm{cm}^{-1}\right): 3060 \mathrm{~m}, 2925 \mathrm{~m}, 1560 \mathrm{vs}, 1471 \mathrm{~s}, 1271 \mathrm{vs}$, 726s, 688m; ${ }^{1} \mathrm{H}$ NMR (300 MHz, $\left.\mathrm{CDCl}_{3}\right): \delta 11.96$ (s, 1H, NH), 7.88-7.85 (m, 2H, PhH), 7.36$7.32(\mathrm{~m}, 3 \mathrm{H}, \mathrm{PhH}), 5.87-5.80\left(\mathrm{~m}, 1 \mathrm{H}, \mathrm{CH}_{2}=\mathrm{CHCH}_{2}\right), 5.65(\mathrm{~s}, 1 \mathrm{H},=\mathrm{CH}), 5.31-5.14(\mathrm{~m}, 2 \mathrm{H}$, $\left.=\mathrm{CH}_{2}\right), 3.94-3.91\left(\mathrm{~m}, 2 \mathrm{H}, \mathrm{NHCH}_{2}\right), 2.31\left(\mathrm{~s}, 3 \mathrm{H}, \mathrm{SCH}_{3}\right) \mathrm{ppm} ;{ }^{13} \mathrm{C} \mathrm{NMR}\left(75 \mathrm{MHz}, \mathrm{CDCl}_{3}\right): \delta$ 185.0, 169.5, 140.6, 132.9, 130.5, 128.2, 126.9, 116.8, 86.6, 46.0, 14.2 ppm; HRMS (EI) Calcd for $\mathrm{C}_{13} \mathrm{H}_{15} \mathrm{NOS}[\mathrm{M}]^{+}$233.0874, Found 233.0878.

(E)-3-(Cyclohexylamino)-3-(methylthio)-1-phenylprop-2-en-1-one (3m). The known compound $\mathbf{3 m}$ was made according to the reported method. ${ }^{13}$ Reaction of $S, S$-acetal $\mathbf{1}\left(\mathrm{R}^{1}=\mathrm{Ph}\right.$, $1.31 \mathrm{~g}, 5.9 \mathrm{mmol})$ and cyclohexylamine $(11.8 \mathrm{mmol})$ generated the product $3 \mathrm{~m}(1.45 \mathrm{~g}, 90 \%)$ as a yellow solid. m.p. $96-97^{\circ} \mathrm{C}$ (EtOAc); FT-IR (film/cm $\left.{ }^{-1}\right): 3060 \mathrm{w}, 2931 \mathrm{~s}, 2852 \mathrm{~m}, 1557 \mathrm{~s}, 1469 \mathrm{~s}$, 1283s, 1066m, 722s, 643m; ${ }^{1} \mathrm{H}$ NMR (300 MHz, $\left.\mathrm{CDCl}_{3}\right): \delta 12.01(\mathrm{~d}, J=8.4 \mathrm{~Hz}, 1 \mathrm{H}, \mathrm{NH}), 7.86-$ $7.83(\mathrm{~m}, 2 \mathrm{H}, \mathrm{PhH}), 7.38-7.34(\mathrm{~m}, 3 \mathrm{H}, \mathrm{PhH}), 5.61(\mathrm{~s}, 1 \mathrm{H},=\mathrm{CH}), 3.62-3.58(\mathrm{~m}, 1 \mathrm{H}, \mathrm{NHC} \underline{\mathrm{H}}), 2.40$ $\left(\mathrm{s}, 3 \mathrm{H}, \mathrm{SCH}_{3}\right), 1.99-1.96(\mathrm{~m}, 2 \mathrm{H}), 1.78-1.75(\mathrm{~m}, 2 \mathrm{H}), 1.58-1.28(\mathrm{~m}, 6 \mathrm{H}) \mathrm{ppm} ;{ }^{13} \mathrm{C} \mathrm{NMR}(75$ $\mathrm{MHz}_{\mathrm{CDCl}}$ ): $\delta 184.8,168.0,140.8,130.3,128.2,126.8,85.9,52.8,33.1,25.4,24.3$, 14.2 ppm; LRMS (ESI, positive) $\mathrm{m} / \mathrm{z} 276.2[\mathrm{M}+\mathrm{H}]^{+}$; Anal.calcd for $\mathrm{C}_{16} \mathrm{H}_{21} \mathrm{NOS}$ : C, 69.78; H, 7.69; N, 5.09. Found: C, 69.72; H, 7.65; N, 5.38. (lit ${ }^{13}$ : no data reported)

(E)-3-(Methylthio)-3-(phenylamino)-1-p-tolylprop-2-en-1-one (3n). The known compound 3n was made according to the reported method. ${ }^{11}$ Reaction of $S, S$-acetal $\mathbf{1}\left(\mathrm{R}_{1}=p-\mathrm{CH}_{3}-\mathrm{Ph}, 1.11 \mathrm{~g}\right.$, $4.7 \mathrm{mmol})$ and aniline $(14.2 \mathrm{mmol})$ generated the product $3 \mathrm{n}(608 \mathrm{mg}, 60 \%)$ as a yellow oil. FTIR (film/ $\mathrm{cm}^{-1}$ ): 3028w, 2923w, 1557vs, 1468s, 1261s, 758s, 693m; $\left.{ }^{1} \mathrm{H} \mathrm{NMR} \mathrm{(300} \mathrm{MHz,} \mathrm{CDCl}_{3}\right)$ : $\delta 13.54(\mathrm{~s}, 1 \mathrm{H}, \mathrm{NH}), 7.83(\mathrm{~d}, J=8.1 \mathrm{~Hz}, 2 \mathrm{H}, \mathrm{PhH}), 7.33-7.28(\mathrm{~m}, 4 \mathrm{H}, \mathrm{PhH}), 7.22-7.20(\mathrm{~m}, 3 \mathrm{H}$, $\mathrm{PhH}), 5.86(\mathrm{~s}, 1 \mathrm{H},=\mathrm{CH}), 2.36\left(\mathrm{~s}, 6 \mathrm{H}, \mathrm{PhCH}_{3}, \mathrm{SCH}_{3}\right) \mathrm{ppm} ;{ }^{13} \mathrm{C} \mathrm{NMR}\left(75 \mathrm{MHz}, \mathrm{CDCl}_{3}\right): \delta 186.0$, 167.1, 141.4, 138.4, 137.5, 129.1, 129.1, 127.2, 126.3, 125.3, 125.2, 88.8, 21.5, 14.8 ppm. [lit ${ }^{11}$ : ${ }^{1} \mathrm{H} \mathrm{NMR}\left(100 \mathrm{MHz}, \mathrm{CDCl}_{3}\right): \delta 13.48(\mathrm{~s}, 1 \mathrm{H}, \mathrm{NH}), 7.81$ (d, $\left.J=8.3 \mathrm{~Hz}, 2 \mathrm{H}, \mathrm{PhH}\right), 7.37-7.25$ (m, $7 \mathrm{H}, \mathrm{PhH}), 5.88$ (s, 1H, 2-H), 2.43 (s, 6H, $\left.\left.\mathrm{PhCH}_{3}, \mathrm{SCH}_{3}\right) \mathrm{ppm}.\right]$

(E)-1-(4-Chlorophenyl)-3-(methylthio)-3-(phenylamino)prop-2-en-1-one (3o). The known compound 3o was made according to the reported method. ${ }^{11}$ Reaction of $S, S$-acetal $1\left(\mathrm{R}^{1}=p\right.$-Cl- 
$\mathrm{Ph}, 2.02 \mathrm{~g}, 7.8 \mathrm{mmol})$ and aniline $(23.4 \mathrm{mmol})$ generated the product $30(1.71 \mathrm{~g}, 72 \%)$ as a yellow solid. m.p. $83-84^{\circ} \mathrm{C}$ (EtOAc); FT-IR (film/ $\mathrm{cm}^{-1}$ ): $3061 \mathrm{w}, 2925 \mathrm{w}, 1557 \mathrm{~s}, 1466 \mathrm{~s}, 1300 \mathrm{~s}$, 1260s, 1092s, 1012s, 839m, 760s, 693m; ${ }^{1} \mathrm{H}$ NMR (300 MHz, $\left.\mathrm{CDCl}_{3}\right): \delta 13.56(\mathrm{~s}, 1 \mathrm{H}, \mathrm{NH})$, 7.83-7.80 (m, 2H, PhH), 7.36-7.18 (m, 7H, PhH), 5.78 (s, 1H, =CH), 2.32 (s, 3H, $\left.\mathrm{SCH}_{3}\right)$ ppm; ${ }^{13} \mathrm{C}$ NMR (75 MHz, $\left.\mathrm{CDCl}_{3}\right): \delta 184.4,168.0,138.6,138.1,137.0,129.1,128.6,126.5,125.1$, 88.5, 14.8 ppm; LRMS (ESI, positive) $\mathrm{m} / \mathrm{z} 304.1[\mathrm{M}+\mathrm{H}]^{+}$; Anal.calcd for $\mathrm{C}_{16} \mathrm{H}_{14} \mathrm{ClNOS}$ : C, 63.25; H, 4.64; N, 4.61. Found: C, 63.31; H, 4.68; N, 4.91. [lit ${ }^{4}$ : m.p. 84 ${ }^{\circ} \mathrm{C} ;{ }^{1} \mathrm{H} \mathrm{NMR}(100 \mathrm{MHz}$, $\left.\mathrm{CDCl}_{3}\right): \delta 13.42(\mathrm{~s}, 1 \mathrm{H}, \mathrm{NH}), 7.92(\mathrm{~d}, J=8.9 \mathrm{~Hz}, 2 \mathrm{H}, \mathrm{PhH}), 7.52-7.23(\mathrm{~m}, 7 \mathrm{H}, \mathrm{PhH}), 6.21(\mathrm{~s}$, $1 \mathrm{H}, 2-\mathrm{H}), 2.52\left(\mathrm{~s}, 3 \mathrm{H}, \mathrm{SCH}_{3}\right) \mathrm{ppm}$.]

(E)-1-(4-Fluorophenyl)-3-(methylthio)-3-(phenylamino)prop-2-en-1-one (3p). Using the general procedure, reaction of $S, S$-acetal $1\left(\mathrm{R}^{1}=p\right.$-F-Ph, $\left.1.27 \mathrm{~g}, 5.3 \mathrm{mmol}\right)$ and aniline (15.9 mmol) generated the product $\mathbf{3 p}(1.16 \mathrm{~g}, 76 \%)$ as a yellow solid. m.p. $70-71^{\circ} \mathrm{C}$ (EtOAc); FT-IR (film/cm $\mathrm{cm}^{-1}$ ): 3062w, 2925w, 1557s, 1468s, 1259s, 1155s, 848m, 767m, 693m; ${ }^{1} \mathrm{H}$ NMR $(300$ $\left.\mathrm{MHz} \mathrm{CDCl}_{3}\right): \delta 13.49$ (s, 1H, NH), 7.93-7.88 (m, 2H, PhH), 7.37-7.19 (m, 5H, PhH), 7.10-7.05 $(\mathrm{m}, 2 \mathrm{H}, \mathrm{PhH}), 5.81(\mathrm{~s}, 1 \mathrm{H},=\mathrm{CH}), 2.37\left(\mathrm{~s}, 3 \mathrm{H}, \mathrm{SCH}_{3}\right) \mathrm{ppm} ;{ }^{13} \mathrm{C} \mathrm{NMR}\left(75 \mathrm{MHz}, \mathrm{CDCl}_{3}\right): \delta 184.6$, 167.7, 166.2 and $164.6\left(\mathrm{~d},{ }^{1} J_{\mathrm{C}-\mathrm{F}}=249.8 \mathrm{~Hz}, 1 \mathrm{C}, \mathrm{C}-\mathrm{F}\right), 138.2,136.4\left(\mathrm{~d},{ }^{4} J_{\mathrm{C}-\mathrm{F}}=3.0 \mathrm{~Hz}, 1 \mathrm{C}\right)$, $129.3\left(\mathrm{~d},{ }^{3} J_{\mathrm{C}-\mathrm{F}}=9.0 \mathrm{~Hz}, 2 \mathrm{C}\right), 129.1,126.5,125.2,115.3\left(\mathrm{~d},{ }^{2} J_{\mathrm{C}-\mathrm{F}}=21.8 \mathrm{~Hz}, 2 \mathrm{C}\right), 88.4,11.7 \mathrm{ppm}$; LRMS (ESI, positive) $\mathrm{m} / z 288.1[\mathrm{M}+\mathrm{H}]^{+}$; Anal.calcd for $\mathrm{C}_{16} \mathrm{H}_{14} \mathrm{FNOS}$ : C, 66.88; H, 4.91; N, 4.87. Found: C, 67.08; H, 4.93; N, 5.07.

(E)-3-(Cyclohexylamino)-3-(methylthio)-1-p-tolylprop-2-en-1-one (3q). The known compound $\mathbf{3 q}$ was made according to the reported method. ${ }^{13}$ Reaction of $S, S$-acetal $\mathbf{1}\left(\mathrm{R}^{1}=p\right.$ $\left.\mathrm{CH}_{3}-\mathrm{Ph}, 1.04 \mathrm{~g}, 4.4 \mathrm{mmol}\right)$ and cyclohexylamine $(8.8 \mathrm{mmol})$ generated the product $\mathbf{3 q}(1.26 \mathrm{~g}$, $85 \%$ ) as a yellow oil. FT-IR (film/ $\mathrm{cm}^{-1}$ ): 2930s, 2853m, 1559vs, $1470 \mathrm{~s}, 1268 \mathrm{~s}, 1080 \mathrm{~m}, 755 \mathrm{~m} ;{ }^{1} \mathrm{H}$ NMR (300 MHz, $\left.\mathrm{CDCl}_{3}\right): \delta 12.00(\mathrm{~d}, J=8.4 \mathrm{~Hz}, 1 \mathrm{H}, \mathrm{NH}), 7.77-7.74(\mathrm{~m}, 2 \mathrm{H}, \mathrm{PhH}), 7.17-7.14$ $(\mathrm{m}, 2 \mathrm{H}, \mathrm{PhH}), 5.59(\mathrm{~s}, 1 \mathrm{H},=\mathrm{CH}), 3.61-3.57(\mathrm{~m}, 1 \mathrm{H}, \mathrm{NHC} \underline{\mathrm{H}}), 2.36\left(\mathrm{~s}, 3 \mathrm{H}, \mathrm{PhCH}_{3}\right), 2.32(\mathrm{~s}, 3 \mathrm{H}$, $\left.\mathrm{SCH}_{3}\right), 1.98-1.94(\mathrm{~m}, 2 \mathrm{H}), 1.76-1.74(\mathrm{~m}, 2 \mathrm{H}), 1.57-1.25(\mathrm{~m}, 6 \mathrm{H}) \mathrm{ppm} ;{ }^{13} \mathrm{C} \mathrm{NMR}(75 \mathrm{MHz}$, $\left.\mathrm{CDCl}_{3}\right): \delta 184.7,167.7,140.5,138.0,130.3,128.8,126.8,85.7,52.7,33.2,25.4,24.3,21.3,14.2$ ppm. (lit ${ }^{13}$ : no data reported)

(E)-1-(4-Chlorophenyl)-3-(cyclohexylamino)-3-(methylthio)prop-2-en-1-one (3r). The known compound $3 \mathbf{r}$ was made according to the reported method. ${ }^{13}$ Reaction of $S, S$-acetal $\mathbf{1}\left(\mathrm{R}^{1}\right.$ $=p$-Cl-Ph, $1.58 \mathrm{~g}, 6.1 \mathrm{mmol})$ and cyclohexylamine $(12.2 \mathrm{mmol})$ generated the product $3 \mathrm{r}(1.88 \mathrm{~g}$, $98 \%$ ) as a yellow oil. FT-IR (film/ $\mathrm{cm}^{-1}$ ): 2931s, 2853m, 1558s, 1470s, 1288s, 1091s, $1012 \mathrm{~m}$, 845m, 763m; ${ }^{1} \mathrm{H}$ NMR (300 MHz, $\left.\mathrm{CDCl}_{3}\right): \delta 12.02(\mathrm{~d}, J=8.7 \mathrm{~Hz}, 1 \mathrm{H}, \mathrm{NH}), 7.79-7.76(\mathrm{~m}, 2 \mathrm{H}$, $\mathrm{PhH}), 7.37-7.31(\mathrm{~m}, 2 \mathrm{H}, \mathrm{PhH}), 5.54(\mathrm{~s}, 1 \mathrm{H},=\mathrm{CH}), 3.62-3.57(\mathrm{~m}, 1 \mathrm{H}, \mathrm{NHC} \underline{\mathrm{H}}), 2.38(\mathrm{~s}, 3 \mathrm{H}$, $\left.\mathrm{SCH}_{3}\right), 1.97-1.94(\mathrm{~m}, 2 \mathrm{H}), 1.76-1.74(\mathrm{~m}, 2 \mathrm{H}), 1.57-1.25(\mathrm{~m}, 6 \mathrm{H}) \mathrm{ppm} ;{ }^{13} \mathrm{C} \mathrm{NMR}(75 \mathrm{MHz}$, $\left.\mathrm{CDCl}_{3}\right): \delta$ 183.0, 168.4, 139.1, 136.2, 128.2, 128.2, 85.6, 52.8, 33.0, 25.3, 24.2, 14.1 ppm; HRMS (EI) Calcd for $\mathrm{C}_{16} \mathrm{H}_{20} \mathrm{CINOS}[\mathrm{M}]^{+} 309.0954$, Found 309.0958. (lit ${ }^{13}$ : no data reported)

(E)-3-(Cyclohexylamino)-1-(4-methoxyphenyl)-3-(methylthio)prop-2-en-1-one (3s). The known compound 3s was made according to the reported method. ${ }^{13}$ Reaction of $S, S$-acetal 1 ( $\mathrm{R}^{1}$ $\left.=p-\mathrm{CH}_{3} \mathrm{O}-\mathrm{Ph}, 1.20 \mathrm{~g}, 4.7 \mathrm{mmol}\right)$ and cyclohexylamine $(9.4 \mathrm{mmol})$ generated the product $3 \mathrm{~s}$ 
$(1.23 \mathrm{~g}, 88 \%)$ as a white solid. m.p. $76-78^{\circ} \mathrm{C}$ (EtOAc); FT-IR (film/ $\left./ \mathrm{cm}^{-1}\right): 2930 \mathrm{~m}, 2852 \mathrm{~m}$, 1560s, 1470s, 1252s, 1169m, 1031m; ${ }^{1} \mathrm{H}$ NMR (300 MHz, $\left.\mathrm{CDCl}_{3}\right): \delta 11.96(\mathrm{~d}, J=8.4 \mathrm{~Hz}, 1 \mathrm{H}$, $\mathrm{NH}), 7.84(\mathrm{~d}, J=9.0 \mathrm{~Hz}, 2 \mathrm{H}, \mathrm{PhH}), 6.87(\mathrm{~d}, J=9.0 \mathrm{~Hz}, 2 \mathrm{H}, \mathrm{PhH}), 5.58(\mathrm{~s}, 1 \mathrm{H},=\mathrm{CH}), 3.74(\mathrm{~s}$, $\left.3 \mathrm{H}, \mathrm{OCH}_{3}\right), 3.60-3.58(\mathrm{~m}, 1 \mathrm{H}, \mathrm{NHC} \underline{\mathrm{H}}), 2.38\left(\mathrm{~s}, 3 \mathrm{H}, \mathrm{SCH}_{3}\right), 1.97-1.94(\mathrm{~m}, 2 \mathrm{H}), 1.76-1.74(\mathrm{~m}$, 2H), 1.56-1.30 (m, 6H) ppm; ${ }^{13} \mathrm{C}$ NMR (75 MHz, $\left.\mathrm{CDCl}_{3}\right): \delta 184.0,167.3,161.5,133.2,128.5$, 113.3, 85.3, 55.1, 52.6, 33.1, 25.4, 24.2, 14.1 ppm; LRMS (ESI, positive) $m / z 306.2[\mathrm{M}+\mathrm{H}]^{+}$; Anal.calcd for $\mathrm{C}_{17} \mathrm{H}_{23} \mathrm{NO}_{2} \mathrm{~S}$ : C, 66.85; H, 7.59; N, 4.59. Found: C, 66.68; H, 7.60; N, 4.72. (lit ${ }^{13}$ : no data reported)

\section{General procedure for the synthesis of $\mathrm{BF}_{\mathbf{2}}$ complex from $\mathbf{N}, \mathrm{S}$-acetal}

To a stirring mixture of ketene $N, S$-acetal 3 (1.0 equiv) and $\mathrm{Et}_{3} \mathrm{~N}$ (3.0 equiv) in dry $\mathrm{MeCN}$ (20 $\mathrm{mL}$ ) was added $\mathrm{BF}_{3} \cdot \mathrm{Et}_{2} \mathrm{O}$ (5.0 equiv) at room temperature. The reaction was monitored by TLC and after TLC indicated the completion of the reaction, the reaction mixture was quenched by addition of saturated aq. $\mathrm{NaHCO}_{3}(30 \mathrm{~mL})$ and extracted with $\mathrm{CH}_{2} \mathrm{Cl}_{2}(3 \times 20 \mathrm{~mL})$. The combined organic layers were dried over $\mathrm{Na}_{2} \mathrm{SO}_{4}$, filtered and concentrated. The resulting precipitates were crystallized from a mixture of ethyl acetate and petroleum ether $(\mathrm{v} / \mathrm{v}=1 / 5-1 / 10)$ to afford pure $\mathrm{BF}_{2}$ complexes 4 .

1-Difluoroboronyloxyl-3-methylthio-1-phenyl-3-( $N$-propylimino)-1-propene (4a). Following the general procedure, reaction of $N, S$-acetal 3a $(300 \mathrm{mg}, 1.28 \mathrm{mmol})$ yielded the product $4 \mathbf{a}$ (323 mg, 88\%) as a white solid. m.p. $134-135^{\circ} \mathrm{C}$ (EtOAc); FT-IR (film/ $/ \mathrm{cm}^{-1}$ ): $2962 \mathrm{w}, 2930 \mathrm{w}$, 2874w, 1597m, 1574m, 1521s, 1500m, 1459m, 1101s, 1022s, 750s, 686m; ${ }^{1} \mathrm{H} \mathrm{NMR} \mathrm{(300} \mathrm{MHz,}$ $\left.\mathrm{CDCl}_{3}\right): \delta 7.89-7.86(\mathrm{~m}, 2 \mathrm{H}, \mathrm{PhH}), 7.36-7.39(\mathrm{~m}, 3 \mathrm{H}, \mathrm{PhH}), 6.07(\mathrm{~s}, 1 \mathrm{H},=\mathrm{CH}), 3.60(\mathrm{t}, J=5.6$ $\left.\mathrm{Hz}, 2 \mathrm{H}, \mathrm{NCH}_{2}\right), 2.60\left(\mathrm{~s}, 3 \mathrm{H}, \mathrm{SCH}_{3}\right), 1.83-1.76\left(\mathrm{~m}, 2 \mathrm{H}, \mathrm{CH}_{2}\right), 0.99(\mathrm{t}, J=7.4 \mathrm{~Hz}, 3 \mathrm{H}) \mathrm{ppm} ;{ }^{13} \mathrm{C}$ NMR (75 MHz, $\left.\mathrm{CDCl}_{3}\right): \delta 174.1,167.1,133.6,132.0,128.6,127.2,89.8,49.4,21.9,14.8,11.5$ ppm; LRMS (ESI, negative) $\mathrm{m} / z 268.2$ [M-15]'; Anal.calcd for $\mathrm{C}_{13} \mathrm{H}_{16} \mathrm{BF}_{2} \mathrm{NOS}$ : C, 55.14; $\mathrm{H}$, 5.70; N, 4.95. Found: C, 55.23; H, 5.82; N, 4.95 .

1-Difluoroboronyloxyl-3-methylthio-3-( $N$-propylimino)-1-p-tolyl-1-propene (4b). Following the general procedure, reaction of $N, S$-acetal $\mathbf{3 b}(350 \mathrm{mg}, 1.41 \mathrm{mmol})$ yielded the product $\mathbf{4 b}$ (402 mg, 95\%) as a white solid. m.p. $126-127^{\circ} \mathrm{C}$ (EtOAc); FT-IR (film/ $/ \mathrm{cm}^{-1}$ ): $2975 \mathrm{w}, 2874 \mathrm{w}$, $1559 \mathrm{~m}, 1519 \mathrm{~s}, 1499 \mathrm{~s}, 1469 \mathrm{~m}, 1102 \mathrm{~m}, 1012 \mathrm{~s}, 766 \mathrm{~s} ;{ }^{1} \mathrm{H} \mathrm{NMR}\left(300 \mathrm{MHz}, \mathrm{CDCl}_{3}\right): \delta 7.78-7.75$ (m, 2H, PhH), 7.21-7.19 (m, 2H, PhH), $6.03(\mathrm{~s}, 1 \mathrm{H},=\mathrm{CH}), 3.59\left(\mathrm{t}, J=5.6 \mathrm{~Hz}, 2 \mathrm{H}, \mathrm{NCH}_{2}\right), 2.59$ $\left(\mathrm{s}, 3 \mathrm{H}, \mathrm{PhCH}_{3}\right), 2.37\left(\mathrm{~s}, 3 \mathrm{H}, \mathrm{SCH}_{3}\right), 1.82-1.75\left(\mathrm{~m}, 2 \mathrm{H}, \mathrm{CH}_{2}\right), 0.98(\mathrm{t}, J=7.4 \mathrm{~Hz}, 3 \mathrm{H}) \mathrm{ppm} ;{ }^{13} \mathrm{C}$ NMR (75 MHz, $\left.\mathrm{CDCl}_{3}\right): \delta 173.9,167.3,142.7,130.8,129.4,127.2,89.3,49.4,22.0,21.5,14.8$, 11.5 ppm; LRMS (ESI, negative) $m / z$ 282.1 [M-15]' Anal.calcd for $\mathrm{C}_{14} \mathrm{H}_{18} \mathrm{BF}_{2} \mathrm{NOS}$ : C, 56.58; H, 6.11; N, 4.71. Found: C, 56.53; H, 6.17; N, 4.83.

1-(4-Chlorophenyl)-1-difluoroboronyloxyl-3-methylthio-3-( $N$-propylimino)-1-propene (4c). Following the general procedure, reaction of $N, S$-acetal $3 \mathrm{c}(322 \mathrm{mg}, 1.20 \mathrm{mmol})$ yielded the product 4c (375 mg, 98\%) as a white solid. m.p. $138-139^{\circ} \mathrm{C}$ (EtOAc); FT-IR (film/ $\left.\mathrm{cm}^{-1}\right): 3018 \mathrm{w}$, 2960w, 2879w, 1599s, 1566m, 1521s, 1487m, 1087s, 1023s, 769m; ${ }^{1} \mathrm{H}$ NMR (300 MHz, 
$\left.\mathrm{CDCl}_{3}\right): \delta 7.81-7.78(\mathrm{~m}, 2 \mathrm{H}, \mathrm{PhH}), 7.38-7.35(\mathrm{~m}, 2 \mathrm{H}, \mathrm{PhH}), 6.04(\mathrm{~s}, 1 \mathrm{H},=\mathrm{CH}), 3.60(\mathrm{t}, J=5.5$ $\left.\mathrm{Hz}, 2 \mathrm{H}, \mathrm{NCH}_{2}\right), 2.61\left(\mathrm{~s}, 3 \mathrm{H}, \mathrm{SCH}_{3}\right), 1.82-1.75\left(\mathrm{~m}, 2 \mathrm{H}, \mathrm{CH}_{2}\right), 0.99(\mathrm{t}, J=7.4 \mathrm{~Hz}, 3 \mathrm{H}) \mathrm{ppm} ;{ }^{13} \mathrm{C}$ NMR $\left(75 \mathrm{MHz}, \mathrm{CDCl}_{3}\right): \delta 174.2,165.7,138.2,132.0,128.9,128.4,89.9,49.5,21.9,14.8,11.4$ ppm; LRMS (ESI, negative) $m / z$ 302.1 [M-15]; Anal.calcd for $\mathrm{C}_{13} \mathrm{H}_{15} \mathrm{BClF}_{2} \mathrm{NOS}$ : C, 49.16; $\mathrm{H}$, 4.76; N, 4.41. Found: C, 49.25; H, 4.85; N, 4.31.

\section{1-Difluoroboronyloxyl-1-(4-methoxyphenyl)-3-methylthio-3-( $N$-propylimino)-1-propene}

(4d). Following the general procedure, reaction of $N, S$-acetal 3d (330 $\mathrm{mg}, 1.25 \mathrm{mmol})$ yielded the product 4d $(340 \mathrm{mg}, 87 \%)$ as a yellow solid. m.p. $132-133^{\circ} \mathrm{C}\left(\right.$ EtOAc); FT-IR (film $\left./ \mathrm{cm}^{-1}\right)$ : $2967 \mathrm{w}, 2836 \mathrm{w}, 1590 \mathrm{~s}, 1521 \mathrm{~s}, 1498 \mathrm{~s}, 1467 \mathrm{~m}, 1256 \mathrm{~s}, 1100 \mathrm{~s}, 1024 \mathrm{~s} ;{ }^{1} \mathrm{H} \mathrm{NMR}\left(300 \mathrm{MHz}, \mathrm{CDCl}_{3}\right)$ : $\delta$ 7.86-7.83 (m, 2H, PhH), 6.92-6.89 (m, 2H, PhH), $5.98(\mathrm{~s}, 1 \mathrm{H},=\mathrm{CH}), 3.84\left(\mathrm{~s}, 3 \mathrm{H}, \mathrm{OCH}_{3}\right), 3.59$ (t, $\left.J=6.2 \mathrm{~Hz}, 2 \mathrm{H}, \mathrm{NCH}_{2}\right), 2.59\left(\mathrm{~s}, 3 \mathrm{H}, \mathrm{SCH}_{3}\right), 1.83-1.75\left(\mathrm{~m}, 2 \mathrm{H}, \mathrm{CH}_{2}\right), 0.99(\mathrm{t}, J=7.4 \mathrm{~Hz}, 3 \mathrm{H})$ ppm; ${ }^{13} \mathrm{C}$ NMR $\left(75 \mathrm{MHz}, \mathrm{CDCl}_{3}\right): \delta 173.6,167.0,162.9,129.1,125.8,114.0,88.6,55.4,49.3$, 22.0, 14.8, 11.5 ppm; LRMS (ESI, negative) $\mathrm{m} / \mathrm{z} 298.2$ [M-15]; Anal.calcd for $\mathrm{C}_{14} \mathrm{H}_{18} \mathrm{BF}_{2} \mathrm{NO}_{2} \mathrm{~S}$ : C, 53.69; H, 5.79; N, 4.47. Found: C, 53.93; H, 5.90; N, 4.35.

1-(4-tert-Butyl-2,6-dimethyl-3,5-dinitrophenyl)-1-difluoroboronyloxyl-3-methylthio-3-( $\mathrm{N}$ propylimino)-1-propene (4e). Following the general procedure, reaction of $N, S$-acetal $3 \mathbf{e}(334$ $\mathrm{mg}, 0.82 \mathrm{mmol})$ yielded the product $4 \mathrm{e}(332 \mathrm{mg}, 89 \%)$ as a white solid. m.p. $146-147^{\circ} \mathrm{C}$ (EtOAc); FT-IR (film/cm ${ }^{-1}$ ): 2973m, 2935w, 2878w, 1603s, 1539s, 1353s, 1120s, 1045s; ${ }^{1} \mathrm{H}$ NMR $\left(300 \mathrm{MHz}, \mathrm{CDCl}_{3}\right): \delta 5.63(\mathrm{~s}, 1 \mathrm{H},=\mathrm{CH}), 3.84\left(\mathrm{~s}, 3 \mathrm{H}, \mathrm{OCH}_{3}\right), 3.67(\mathrm{t}, J=7.6 \mathrm{~Hz}, 2 \mathrm{H}$, $\left.\mathrm{NCH}_{2}\right), 2.56$ (s, 3H, $\left.\mathrm{SCH}_{3}\right), 2.20-2.17\left(\mathrm{~m}, 6 \mathrm{H}, \mathrm{PhCH}_{3}\right), 1.87-1.79\left(\mathrm{~m}, 2 \mathrm{H}, \mathrm{CH}_{2}\right), 1.45-1.42$ (m, $\left.9 \mathrm{H}, 3 \mathrm{CH}_{3}\right), 1.03(\mathrm{t}, J=7.4 \mathrm{~Hz}, 3 \mathrm{H}) \mathrm{ppm} ;{ }^{13} \mathrm{C} \mathrm{NMR}\left(75 \mathrm{MHz}, \mathrm{CDCl}_{3}\right): \delta 174.4,166.1,150.3$, 136.7, 132.6, 130.5, 96.0, 50.0, 37.5, 30.3, 21.7, 15.5, 14.9, 11.4 ppm; LRMS (ESI, negative) $\mathrm{m} / z$ 442.2 [M-15]; Anal.calcd for $\mathrm{C}_{19} \mathrm{H}_{26} \mathrm{BF}_{2} \mathrm{~N}_{3} \mathrm{O}_{5} \mathrm{~S}: \mathrm{C}, 49.90 ; \mathrm{H}, 5.73 ; \mathrm{N}, 9.19$. Found: C, 49.98; $\mathrm{H}$, $5.81 ; \mathrm{N}, 9.03$.

1-Difluoroboronyloxyl-3-methylthio-1-(4-phenylphenyl)-3-( $N$-propylimino)-1-propene (4f). Following the general procedure, reaction of $N, S$-acetal $3 f(377 \mathrm{mg}, 1.21 \mathrm{mmol})$ yielded the product 4f (395 mg, 91\%) as a yellow solid. m.p. 141-142 ${ }^{\circ} \mathrm{C}$ (EtOAc); FT-IR (film/ $\left./ \mathrm{cm}^{-1}\right)$ : 3033w, 2966m, 2932w, 2874w, 1590s, 1507s, 1103s, 1035s, 758s, 698m; ${ }^{1} \mathrm{H}$ NMR (300 MHz, $\left.\mathrm{CDCl}_{3}\right): \delta$ 7.95-7.93 (m, 2H, PhH), 7.64-7.58 (m, 4H, PhH), 7.46-7.24 (m, 3H, PhH), $6.10(\mathrm{~s}$, $1 \mathrm{H},=\mathrm{CH}), 3.61\left(\mathrm{t}, J=6.7 \mathrm{~Hz}, 2 \mathrm{H}, \mathrm{NCH}_{2}\right), 2.60\left(\mathrm{~s}, 3 \mathrm{H}, \mathrm{SCH}_{3}\right), 1.84-1.76\left(\mathrm{~m}, 2 \mathrm{H}, \mathrm{CH}_{2}\right), 0.99(\mathrm{t}$, $\left.J=7.4 \mathrm{~Hz}, 3 \mathrm{H}, \mathrm{CH}_{3}\right) \mathrm{ppm} ;{ }^{13} \mathrm{C} \mathrm{NMR}\left(75 \mathrm{MHz}, \mathrm{CDCl}_{3}\right): \delta 174.0,166.8,144.7,139.8,132.3$, 129.0, 128.2, 127.7, 127.2, 127.1, 89.7, 49.5, 22.0, 14.8, 11.5 ppm; LRMS (ESI, positive) $\mathrm{m} / z$ 339.9 [M-19] ${ }^{+}$; Anal.calcd for $\mathrm{C}_{19} \mathrm{H}_{20} \mathrm{BF}_{2} \mathrm{NOS}$ : C, 63.52; H, 5.61; N, 3.90. Found: C, 63.45; H, $5.63 ; \mathrm{N}, 4.07$.

1-Difluoroboronyloxyl-1-(4-fluorophenyl)-3-methylthio-3-( $N$-propylimino)-1-propene (4g). Following the general procedure, reaction of $N, S$-acetal $3 \mathrm{~g}(373 \mathrm{mg}, 1.47 \mathrm{mmol})$ yielded the product $4 \mathrm{~g}$ (405 mg, 90\%) as a white solid. m.p. $138-139^{\circ} \mathrm{C}$ (EtOAc); FT-IR (film/ $\mathrm{cm}^{-1}$ ): $2961 \mathrm{w}$, $2873 \mathrm{w}, 1605 \mathrm{~m}, 1581 \mathrm{~s}, 1528 \mathrm{~s}, 1501 \mathrm{~s}, 1456 \mathrm{~m}, 1098 \mathrm{~s}, 1036 \mathrm{~s}, 769 \mathrm{~m} ;{ }^{1} \mathrm{H}$ NMR $\left(300 \mathrm{MHz}, \mathrm{CDCl}_{3}\right)$ : $\delta$ 7.89-7.84 (m, 2H, PhH), 7.10-7.04 (m, 2H, PhH), $6.03(\mathrm{~s}, 1 \mathrm{H},=\mathrm{CH}), 3.56(\mathrm{~d}, J=6.2 \mathrm{~Hz}, 2 \mathrm{H}$, $\left.\mathrm{NCH}_{2}\right), 2.60\left(\mathrm{~s}, 3 \mathrm{H}, \mathrm{SCH}_{3}\right), 1.81-1.74\left(\mathrm{~m}, 2 \mathrm{H}, \mathrm{CH}_{2}\right), 0.98\left(\mathrm{t}, J=7.4 \mathrm{~Hz}, 3 \mathrm{H}, \mathrm{CH}_{3}\right) \mathrm{ppm} ;{ }^{13} \mathrm{C}$ 
NMR (75 MHz, $\left.\mathrm{CDCl}_{3}\right): \delta 174.3,165.7,165.0\left(\mathrm{~d},{ }^{1} J_{\mathrm{C}-\mathrm{F}}=252.0 \mathrm{~Hz}, 1 \mathrm{C}, \mathrm{C}-\mathrm{F}\right), 129.7,129.5(\mathrm{~d}$, $\left.{ }^{3} J_{\mathrm{C}-\mathrm{F}}=9.0 \mathrm{~Hz}, 2 \mathrm{C}\right), 115.8\left(\mathrm{~d},{ }^{2} J_{\mathrm{C}-\mathrm{F}}=21.8 \mathrm{~Hz}, 2 \mathrm{C}\right), 89.6,49.3,21.9,14.7,11.4 \mathrm{ppm}$; LRMS (ESI, negative) $m / z 286.2$ [M-15]; Anal.calcd for $\mathrm{C}_{13} \mathrm{H}_{15} \mathrm{BF}_{3} \mathrm{NOS}$ : C, 51.85; H, 5.02; N, 4.65. Found: C, 52.03; H, 5.17; N, 4.68 .

1-Difluoroboronyloxyl-1-(4-(trifluoromethyl)phenyl)-3-methylthio-3-( $\mathrm{N}$-propylimino)-1propene (4h). Following the general procedure, reaction of $N, S$-acetal $\mathbf{3 h}(325 \mathrm{mg}, 1.07 \mathrm{mmol})$ yielded the product $\mathbf{4 h}(351 \mathrm{mg}, 92 \%)$ as a white solid. m.p. 105-106 ${ }^{\circ} \mathrm{C}$ (EtOAc); FT-IR (film/cm $\mathrm{cm}^{-1}$ ): 2972w, 2938w, 2880w, 1601s, 1574s, 1526s, 1510s, 1324s, $1128 \mathrm{~s}, 1067 \mathrm{~s}, 854 \mathrm{~m}$, 779m; ${ }^{1} \mathrm{H}$ NMR $\left(300 \mathrm{MHz}, \mathrm{CDCl}_{3}\right): \delta 7.97-7.95(\mathrm{~m}, 2 \mathrm{H}, \mathrm{PhH}), 7.65-7.62(\mathrm{~m}, 2 \mathrm{H}, \mathrm{PhH}), 6.16(\mathrm{~s}$, $1 \mathrm{H},=\mathrm{CH}), 3.60\left(\mathrm{t}, J=7.0 \mathrm{~Hz}, 2 \mathrm{H}, \mathrm{NCH}_{2}\right), 2.64\left(\mathrm{~s}, 3 \mathrm{H}, \mathrm{SCH}_{3}\right), 1.82-1.74\left(\mathrm{~m}, 2 \mathrm{H}, \mathrm{CH}_{2}\right), 0.99(\mathrm{t}$, $\left.J=7.4 \mathrm{~Hz}, 3 \mathrm{H}, \mathrm{CH}_{3}\right) \mathrm{ppm} ;{ }^{13} \mathrm{C} \mathrm{NMR}\left(75 \mathrm{MHz}, \mathrm{CDCl}_{3}\right): \delta 174.6,164.8,136.9,133.1\left(\mathrm{q},{ }^{2} J_{\mathrm{C}-\mathrm{F}}=\right.$ $\left.32.2 \mathrm{~Hz}, C \mathrm{CF}_{3}\right), 127.4,125.5\left(\mathrm{q},{ }^{3} J_{\mathrm{C}-\mathrm{F}}=3.8 \mathrm{~Hz}, C H \mathrm{CCF}_{3}\right), 120.1$ (q, $\left.{ }^{1} J_{\mathrm{C}-\mathrm{F}}=270.8 \mathrm{~Hz}, \mathrm{CF}_{3}\right), 90.9$, 49.6, 21.7, 14.7, 11.3 ppm; LRMS (ESI, negative) $\mathrm{m} / \mathrm{z} 336.1$ [M-15]; Anal.calcd for $\mathrm{C}_{14} \mathrm{H}_{15} \mathrm{BF}_{5} \mathrm{NOS}$ : C, 47.89; H, 4.31; N, 3.99. Found: C, 47.79; H, 4.37; N, 4.11.

1-Difluoroboronyloxyl-3-methylthio-1-phenyl-3-( $N$-phenylimino)-1-propene (4i). Following the general procedure, reaction of $N, S$-acetal $3 \mathbf{i}(346 \mathrm{mg}, 1.29 \mathrm{mmol})$ yielded the known product 4i (362 mg, 88\%) as a white solid. m.p. $194-196^{\circ} \mathrm{C}$ (EtOAc); FT-IR (film/ $\left./ \mathrm{cm}^{-1}\right): 3061 \mathrm{w}, 1590 \mathrm{~m}$, $1563 \mathrm{~s}, 1454 \mathrm{~s}, 1112 \mathrm{~s}, 1048 \mathrm{~m}, 757 \mathrm{~s}, 706 \mathrm{~m} ;{ }^{1} \mathrm{H} \mathrm{NMR}\left(300 \mathrm{MHz}, \mathrm{CDCl}_{3}\right): \delta 7.98-7.95$ (m, 2H, $\mathrm{PhH}), 7.53-7.39$ (m, 6H, PhH), 7.31-7.24 (m, 2H, PhH), 6.24 (s, 1H, =CH), 2.47 (s, 3H, $\left.\mathrm{SCH}_{3}\right)$ ppm; ${ }^{13} \mathrm{C}$ NMR $\left(75 \mathrm{MHz}, \mathrm{CDCl}_{3}\right): \delta 177.2,169.2,139.7,133.5,132.5,129.3,128.8,128.8$, 127.5, 127.0, 89.9, 15.4 ppm; HRMS (EI) $m / z$ Calcd for $\mathrm{C}_{16} \mathrm{H}_{14} \mathrm{BF}_{2} \mathrm{NOS}[\mathrm{M}]^{+} 317.0857$, Found 317.0862. [lit ${ }^{4}$ : m.p. $196^{\circ} \mathrm{C}$; H NMR (100 MHz, $\left.\mathrm{CDCl}_{3}\right): \delta 7.93-8.04$ (m, 2H, PhH), 7.25-7.58 (m, 8H, $\left.\mathrm{PhH}), 6.24(\mathrm{~s}, 1 \mathrm{H}, 3-\mathrm{H}), 2.52\left(\mathrm{~s}, 3 \mathrm{H}, \mathrm{SCH}_{3}\right) \mathrm{ppm}.\right]$

1-Difluoroboronyloxyl-3-( $\mathrm{N}$-isopropylimino)-3-methylthio-1-phenyl-1-propene

$(4 \mathrm{j})$.

Following the general procedure, reaction of $N, S$-acetal 3j (339 $\mathrm{mg}, 1.44 \mathrm{mmol}$ ) yielded the product $4 \mathbf{j}$ (400 mg, 96\%) as a white solid. m.p. $132-134^{\circ} \mathrm{C}$ (EtOAc); FT-IR (film/ $\mathrm{cm}^{-1}$ ): $2978 \mathrm{w}$, 2938w, 2874w, 1594s, 1569s, 1489s, 1308m, 1101s, 1005s, 753m, 688m; ${ }^{1} \mathrm{H}$ NMR (300 MHz, $\left.\mathrm{CDCl}_{3}\right): \delta 7.89-7.86(\mathrm{~m}, 2 \mathrm{H}, \mathrm{PhH}), 7.50-7.38(\mathrm{~m}, 3 \mathrm{H}, \mathrm{PhH}), 6.08(\mathrm{~s}, 1 \mathrm{H},=\mathrm{CH}), 4.34-4.28(\mathrm{~m}$, $1 \mathrm{H}, \mathrm{NCH}), 2.60\left(\mathrm{~s}, 3 \mathrm{H}, \mathrm{SCH}_{3}\right), 1.48\left(\mathrm{~d}, J=6.6 \mathrm{~Hz}, 6 \mathrm{H}, 2 \mathrm{CH}_{3}\right) \mathrm{ppm} ;{ }^{13} \mathrm{C} \mathrm{NMR}(75 \mathrm{MHz}$, $\left.\mathrm{CDCl}_{3}\right): \delta 173.3,166.6,133.5,131.9,128.6,127.2,89.9,52.9,21.1,21.1,15.3$ ppm; LRMS (ESI, positive) $m / z 264.2$ [M-19] ${ }^{+}$; Anal.calcd for $\mathrm{C}_{13} \mathrm{H}_{16} \mathrm{BF}_{2} \mathrm{NOS}$ : C, 55.14; H, 5.70; N, 4.95. Found: C, 54.88; H, 5.73; N, 5.05 .

3-(N-Butylimino)-1-difluoroboronyloxyl-3-methylthio-1-phenyl-1-propene (4k). Following the general procedure, reaction of $N, S$-acetal $3 \mathbf{k}(373 \mathrm{mg}, 1.50 \mathrm{mmol})$ yielded the product $4 \mathbf{k}$ (399 mg, 90\%) as a white solid. m.p. $101-102^{\circ} \mathrm{C}$ (EtOAc); FT-IR (film/ $\mathrm{cm}^{-1}$ ): $2962 \mathrm{~m} 2934 \mathrm{~m}$, 2872m, 1596s, 1571s, 1520s, 1492s, 1316m, 1101s, 1032s, 751s, 687m; ${ }^{1} \mathrm{H}$ NMR (300 MHz, $\left.\mathrm{CDCl}_{3}\right): \delta 7.89-7.85(\mathrm{~m}, 2 \mathrm{H}, \mathrm{PhH}), 7.47-7.38(\mathrm{~m}, 3 \mathrm{H}, \mathrm{PhH}), 6.07(\mathrm{~s}, 1 \mathrm{H},=\mathrm{CH}), 3.63(\mathrm{t}, J=5.7$ $\left.\mathrm{Hz}, 2 \mathrm{H}, \mathrm{NCH}_{2}\right), 2.59$ (s, 3H, $\left.\mathrm{SCH}_{3}\right), 1.80-1.69\left(\mathrm{~m}, 2 \mathrm{H}, \mathrm{CH}_{2}\right), 1.42-1.35\left(\mathrm{~m}, 2 \mathrm{H}, \mathrm{CH}_{2}\right), 0.98$ (t, $J$ $=7.4 \mathrm{~Hz}, 3 \mathrm{H}) \mathrm{ppm} ;{ }^{13} \mathrm{C} \mathrm{NMR}\left(75 \mathrm{MHz}, \mathrm{CDCl}_{3}\right): \delta 174.0,167.0,133.6,132.0,128.6,127.1$, 
89.8, 47.7, 30.5, 20.4, 14.8, 13.7 ppm; LRMS (ESI, positive) $m / z 278.2$ [M-19] ; Anal.calcd for $\mathrm{C}_{14} \mathrm{H}_{18} \mathrm{BF}_{2} \mathrm{NOS}$ : C, 56.58; H, 6.11; N, 4.71. Found: C, 56.53; H, 6.21; N, 4.63.

3-(N-Allylimino)-1-difluoroboronyloxyl-3-methylthio-1-phenyl-1-propene (4I). Following the general procedure, reaction of $N, S$-acetal 31 (365 mg, $1.57 \mathrm{mmol})$ yielded the product $4 \mathbf{l}(378$ $\mathrm{mg}, 86 \%$ ) as a yellow solid. m.p. $135-137^{\circ} \mathrm{C}\left(\right.$ EtOAc); FT-IR (film/ $\left.\mathrm{cm}^{-1}\right): 1595 \mathrm{~s}, 1571 \mathrm{~s}, 1517 \mathrm{~s}$, $1500 \mathrm{~m}, 1490 \mathrm{~s}, 1312 \mathrm{~m}, 1102 \mathrm{~s}, 1048 \mathrm{~s}, 749 \mathrm{~m}, 687 \mathrm{~m} ;{ }^{1} \mathrm{H}$ NMR $\left(300 \mathrm{MHz}, \mathrm{CDCl}_{3}\right): \delta 7.90-7.87$ $(\mathrm{m}, 2 \mathrm{H}, \mathrm{PhH}), 7.48-7.38(\mathrm{~m}, 3 \mathrm{H}, \mathrm{PhH}), 6.07(\mathrm{~s}, 1 \mathrm{H},=\mathrm{CH}), 5.91-5.80(\mathrm{~m}, 1 \mathrm{H},=\mathrm{CH}), 5.31-5.23$ $\left(\mathrm{m}, 2 \mathrm{H},=\mathrm{CH}_{2}\right), 4.27\left(\mathrm{~s}, 2 \mathrm{H}, \mathrm{NCH}_{2}\right), 2.59\left(\mathrm{~s}, 3 \mathrm{H}, \mathrm{SCH}_{3}\right) \mathrm{ppm} ;{ }^{13} \mathrm{C} \mathrm{NMR}\left(75 \mathrm{MHz}, \mathrm{CDCl}_{3}\right): \delta$ 175.3, 167.5, 133.4, 132.1, 131.5, 128.7, 127.2, 118.8, 89.9, 49.6, 14.9 ppm; LRMS (ESI, negative) $m / z 266.2$ [M-15] ; Anal.calcd for $\mathrm{C}_{13} \mathrm{H}_{14} \mathrm{BF}_{2} \mathrm{NOS}$ : C, 55.54; H, 5.02; N, 4.98. Found: C, 55.59; H, 4.99; N, 5.10.

3-(N-Cyclohexylimino)-1-difluoroboronyloxyl-3-methylthio-1-phenyl-1-propene

(4m). Following the general procedure, reaction of $N, S$-acetal $3 \mathrm{~m}(325 \mathrm{mg}, 1.18 \mathrm{mmol})$ yielded the product $4 \mathrm{~m}$ (354 mg, 93\%) as a slightly yellow solid. m.p. $144-145^{\circ} \mathrm{C}$ (EtOAc); FT-IR (film/cm ${ }^{-}$ $\left.{ }^{1}\right): 3064 \mathrm{~m}, 2933 \mathrm{~s}, 2855 \mathrm{~m}, 1594 \mathrm{~s}, 1568 \mathrm{~s}, 1505 \mathrm{~s}, 1489 \mathrm{~s}, 1309 \mathrm{~m}, 1104 \mathrm{~s}, 1025 \mathrm{~s}, 990 \mathrm{~s}, 752 \mathrm{~s}, 688 \mathrm{~m}$; ${ }^{1} \mathrm{H}$ NMR (300 MHz, $\left.\mathrm{CDCl}_{3}\right): \delta 7.89-7.86(\mathrm{~m}, 2 \mathrm{H}, \mathrm{PhH}), 7.47-7.37(\mathrm{~m}, 3 \mathrm{H}, \mathrm{PhH}), 6.07(\mathrm{~s}, 1 \mathrm{H}$, $=\mathrm{CH}), 3.86-3.85(\mathrm{~m}, 1 \mathrm{H}, \mathrm{NCH}), 2.59\left(\mathrm{~s}, 3 \mathrm{H}, \mathrm{SCH}_{3}\right), 2.00-1.84(\mathrm{~m}, 6 \mathrm{H}), 1.67-1.66(\mathrm{~m}, 1 \mathrm{H})$, 1.32-1.24 (m, 3H) ppm; ${ }^{13} \mathrm{C}$ NMR (75 MHz, $\left.\mathrm{CDCl}_{3}\right): \delta 173.3,166.5,133.5,131.9,128.6,127.1$, 89.8, 61.6, 31.1, 26.1, 25.2, 15.3 ppm; LRMS (ESI, positive) $m / z 304.1$ [M-19] ; Anal.calcd for $\mathrm{C}_{16} \mathrm{H}_{20} \mathrm{BF}_{2} \mathrm{NOS}$ : C, 59.46; H, 6.24; N, 4.33. Found: C, 59.29; H, 6.30; N, 4.37.

1-Difluoroboronyloxyl-3-methylthio-3-( $N$-phenylimino)-1-p-tolyl-1-propene (4n). Following the general procedure, reaction of $N, S$-acetal $3 \mathrm{n}(325 \mathrm{mg}, 1.15 \mathrm{mmol})$ yielded the known product 4n (342 mg, 90\%) as a white solid. m.p. 210-211 ${ }^{\circ} \mathrm{C}$ (EtOAc); FT-IR (film/ $\left./ \mathrm{cm}^{-1}\right): 1585 \mathrm{~m}, 1558 \mathrm{~s}$, 1491s, 1111s, 1038s, 755s, 695m; ${ }^{1} \mathrm{H}$ NMR (300 MHz, $\left.\mathrm{CDCl}_{3}\right): \delta 7.88(\mathrm{~d}, J=8.3 \mathrm{~Hz}, 2 \mathrm{H}, \mathrm{PhH})$, 7.44-7.39 (m, 3H, PhH), 7.31-7.24 (m, 4H, PhH), 6.21 (s, 1H, =CH), $2.48\left(\mathrm{~s}, 3 \mathrm{H}, \mathrm{SCH}_{3}\right), 2.41$ $\left(\mathrm{s}, 3 \mathrm{H}, \mathrm{PhCH}_{3}\right) \mathrm{ppm} ;{ }^{13} \mathrm{C} \mathrm{NMR}\left(75 \mathrm{MHz}, \mathrm{CDCl}_{3}\right): \delta 176.9,169.4,143.4,139.8,130.6,129.5$, 129.3, 128.7, 127.6, 127.1, 89.4, 21.6, 15.3 ppm; HRMS (EI) $m / z$ Calcd for $\mathrm{C}_{17} \mathrm{H}_{16} \mathrm{BF}_{2} \mathrm{NOS}$ [M] ${ }^{+}$ 331.1014, Found 331.1018. [lit ${ }^{4}$ : m.p. $212^{\circ} \mathrm{C}$; ${ }^{1} \mathrm{H}$ NMR $\left(100 \mathrm{MHz}, \mathrm{CDCl}_{3}\right): \delta 7.87(\mathrm{~d}, J=8.4$ $\mathrm{Hz}, 2 \mathrm{H}, \mathrm{PhH}), 7.35$ (d, J=8.4 Hz, 2H, PhH), 7.48-7.21 (m, 5H, PhH), 6.20 (s, 1H, 3-H), 2.49 (s, $\left.3 \mathrm{H}, \mathrm{SCH}_{3}\right), 2.42$ (s, 3H, 4-Me) ppm.]

1-(4-Chlorophenyl)-1-difluoroboronyloxyl-3-methylthio-3-( $N$-phenylimino)-1-propene (4o). Following the general procedure, reaction of $N, S$-acetal 3o (369 $\mathrm{mg}, 1.22 \mathrm{mmol})$ yielded the known product 40 (389 mg, 98\%) as a white solid. m.p. $184-185^{\circ} \mathrm{C}$ (EtOAc); FT-IR (film/ $\left.\mathrm{cm}^{-1}\right)$ : $1586 \mathrm{~s}, 1557 \mathrm{~s}, 1502 \mathrm{~s}, 1482 \mathrm{~s}, 1115 \mathrm{~m}, 1043 \mathrm{~m}, 764 \mathrm{~m}, 696 \mathrm{~m} ;{ }^{1} \mathrm{H}$ NMR $\left(300 \mathrm{MHz}, \mathrm{CDCl}_{3}\right): \delta 7.90$ $(\mathrm{d}, J=8.6 \mathrm{~Hz}, 2 \mathrm{H}, \mathrm{PhH}), 7.47-7.40(\mathrm{~m}, 5 \mathrm{H}, \mathrm{PhH}), 7.29-7.24(\mathrm{~m}, 2 \mathrm{H}, \mathrm{PhH}), 6.21(\mathrm{~s}, 1 \mathrm{H},=\mathrm{CH})$, $2.46\left(\mathrm{~s}, 3 \mathrm{H}, \mathrm{SCH}_{3}\right) \mathrm{ppm} ;{ }^{13} \mathrm{C} \mathrm{NMR}\left(75 \mathrm{MHz}, \mathrm{CDCl}_{3}\right): \delta 177.5,167.7,139.6,138.8,131.9,129.3$, 129.1, 128.8, 126.9, 90.0, 15.3 ppm; LRMS (ESI, positive) $m / z 332.0$ [M-19] ${ }^{+}$; Anal.calcd for $\mathrm{C}_{16} \mathrm{H}_{13} \mathrm{BClF}_{2} \mathrm{NOS}$ : C, 54.66; H, 3.73; N, 3.98. Found: C, 54.68; H, 3.77; N, 4.18. [lit ${ }^{4}$ : m.p. $184^{\circ} \mathrm{C} ;{ }^{1} \mathrm{H}$ NMR $\left(100 \mathrm{MHz}, \mathrm{CDCl}_{3}\right): \delta 7.90(\mathrm{~d}, J=8.8 \mathrm{~Hz}, 2 \mathrm{H}, \mathrm{PhH}), 7.42(\mathrm{~d}, J=8.8 \mathrm{~Hz}, 2 \mathrm{H}$, $\mathrm{PhH}), 7.52-7.22$ (m, 5H, PhH), 6.20 (s, 1H, 3-H), 2.50 (s, 3H, $\left.\left.\mathrm{SCH}_{3}\right) \mathrm{ppm}.\right)$ 
1-Difluoroboronyloxyl-1-(4-fluorophenyl)-3-methylthio-3-( $N$-phenylimino)-1-propene (4p). Following the general procedure, reaction of $N, S$-acetal 3p (349 mg, $1.22 \mathrm{mmol})$ yielded the product 4p (379 mg, 93\%) as a white solid. m.p. 178-179 ${ }^{\circ} \mathrm{C}\left(\right.$ EtOAc); FT-IR (film/ $\left./ \mathrm{cm}^{-1}\right): 1602 \mathrm{~s}$, $1568 \mathrm{~s}, 1490 \mathrm{~s}, 1114 \mathrm{~m}, 1042 \mathrm{~m}, 757 \mathrm{~m}, 696 \mathrm{~m} ;{ }^{1} \mathrm{H}$ NMR $\left(300 \mathrm{MHz}, \mathrm{CDCl}_{3}\right): \delta 8.01-7.97$ (m, 2H, $\mathrm{PhH}), 7.46-7.41$ (m, 3H, PhH), 7.32-7.29 (m, 2H, PhH), 7.18 (t, J=8.6 Hz, 2H, PhH), 6.19 (s, $1 \mathrm{H},=\mathrm{CH}), 2.50\left(\mathrm{~s}, 3 \mathrm{H}, \mathrm{SCH}_{3}\right) \mathrm{ppm} ;{ }^{13} \mathrm{C} \mathrm{NMR}\left(75 \mathrm{MHz}, \mathrm{CDCl}_{3}\right): \delta 177.2,168.0,165.4\left(\mathrm{~d},{ }^{1} J_{\mathrm{C}-\mathrm{F}}\right.$ $=252.8 \mathrm{~Hz}, 1 \mathrm{C}, \mathrm{C}-\mathrm{F}), 139.6,129.9\left(\mathrm{~d},{ }^{3} J_{\mathrm{C}-\mathrm{F}}=9.0 \mathrm{~Hz}, 2 \mathrm{C}\right), 129.6,129.3,128.8,126.9,116.0(\mathrm{~d}$, ${ }^{2} J_{\mathrm{C}-\mathrm{F}}=21.8 \mathrm{~Hz}, 2 \mathrm{C}$ ), 89.5, $15.4 \mathrm{ppm}$; LRMS (ESI, positive) $\mathrm{m} / \mathrm{z} 316.1[\mathrm{M}-19]^{+}$; Anal.calcd for $\mathrm{C}_{16} \mathrm{H}_{13} \mathrm{BF}_{3} \mathrm{NOS}$ : C, 57.34; H, 3.91; N, 4.18. Found: C, 57.42; H, 4.02; N, 4.31.

3-(N-Cyclohexylimino)-1-difluoroboronyloxyl-3-methylthio-1-p-tolyl-1-propene

(4q). Following the general procedure, reaction of $N, S$-acetal $\mathbf{3 q}(435 \mathrm{mg}, 1.51 \mathrm{mmol})$ yielded the product 4q (431 mg, 85\%) as a slightly yellow solid. m.p. $155-156^{\circ} \mathrm{C}$ (EtOAc); FT-IR (film/ $\mathrm{cm}^{-}$ $\left.{ }^{1}\right): 2932 \mathrm{~m}, 2855 \mathrm{~m}, 1590 \mathrm{~s}, 1564 \mathrm{~s}, 1496 \mathrm{~s}, 1489 \mathrm{~s}, 1308 \mathrm{~m}, 1101 \mathrm{~s}, 1005 \mathrm{~s}, 753 \mathrm{~s}, 688 \mathrm{~m}$; ${ }^{1} \mathrm{H}$ NMR $\left(300 \mathrm{MHz}, \mathrm{CDCl}_{3}\right): \delta 7.78(\mathrm{~d}, J=8.2 \mathrm{~Hz}, 2 \mathrm{H}, \mathrm{PhH}), 7.20(\mathrm{~d}, J=8.1 \mathrm{~Hz}, 2 \mathrm{H}, \mathrm{PhH}), 6.03(\mathrm{~s}, 1 \mathrm{H}$, $=\mathrm{CH}), 3.84-3.83(\mathrm{~m}, 1 \mathrm{H}, \mathrm{NCH}), 2.57\left(\mathrm{~s}, 3 \mathrm{H}, \mathrm{SCH}_{3}\right), 2.36\left(\mathrm{~s}, 3 \mathrm{H}, \mathrm{PhCH}_{3}\right), 1.99-1.83(\mathrm{~m}, 6 \mathrm{H})$, 1.67-1.65 (m, 1H), 1.26-1.23 (m, 3H) ppm; $\left.{ }^{13} \mathrm{C} \mathrm{NMR(75} \mathrm{MHz,} \mathrm{CDCl}_{3}\right): \delta 173.2,166.6,142.6$, $130.7,129.3,127.2,89.3,61.5,31.1,26.1,25.2,21.5,15.3 \mathrm{ppm}$; HRMS (EI) $\mathrm{m} / z$ Calcd for $\mathrm{C}_{17} \mathrm{H}_{22} \mathrm{BF}_{2} \mathrm{NOS}[\mathrm{M}]^{+}$337.1483, Found 337.1488.

\section{1-(4-Chlorophenyl)-3-( $N$-cyclohexylimino)-1-difluoroboronyloxyl-3-methylthio-1-propene}

(4r). Following the general procedure, reaction of $N, S$-acetal $3 r(376 \mathrm{mg}, 1.22 \mathrm{mmol})$ yielded the product $4 \mathbf{r}(361 \mathrm{mg}, 83 \%)$ as a white solid. m.p. $173-174^{\circ} \mathrm{C}\left(\right.$ EtOAc); FT-IR (film $\left./ \mathrm{cm}^{-1}\right): 2933 \mathrm{w}$, $2855 \mathrm{w}, 1594 \mathrm{~s}, 1560 \mathrm{~s}, 1485 \mathrm{~s}, 1437 \mathrm{~s}, 1102 \mathrm{~m}, 1024 \mathrm{~m}, 989 \mathrm{~m} ;{ }^{1} \mathrm{H}$ NMR $\left(300 \mathrm{MHz}, \mathrm{CDCl}_{3}\right): \delta$ 7.74-7.80 (m, 2H, PhH), 7.41-7.26 (m, 2H, PhH), $6.04(\mathrm{~s}, 1 \mathrm{H},=\mathrm{CH}), 3.86-3.79(\mathrm{~m}, 1 \mathrm{H}, \mathrm{NCH})$, $2.62\left(\mathrm{~s}, 3 \mathrm{H}, \mathrm{SCH}_{3}\right), 2.01-1.86(\mathrm{~m}, 6 \mathrm{H}), 1.68-1.59(\mathrm{~m}, 1 \mathrm{H}), 1.28-1.25(\mathrm{~m}, 3 \mathrm{H}) \mathrm{ppm} ;{ }^{13} \mathrm{C} \mathrm{NMR}$ $\left(75 \mathrm{MHz}, \mathrm{CDCl}_{3}\right): \delta 173.2,165.4,138.1,132.0,128.9,128.5,89.9,61.9,31.0,26.1,25.1,15.4$ ppm; LRMS (ESI, negative) $\mathrm{m} / z$ 342.0 [M-15]; Anal.calcd for $\mathrm{C}_{16} \mathrm{H}_{19} \mathrm{BClF}_{2} \mathrm{NOS}$ : C, 53.73; $\mathrm{H}$, 5.35; N, 3.92. Found: C, 53.55; H, 5.20; N, 3.89.

\section{3-(N-Cyclohexylimino)-1-difluoroboronyloxyl-1-(4-methoxyphenyl)-3-methylthio-1-}

propene (4s). Following the general procedure, reaction of $N, S$-acetal 3s (375 $\mathrm{mg}, 1.23 \mathrm{mmol})$ yielded the product $4 \mathbf{s}(321 \mathrm{mg}, 74 \%)$ as a slightly yellow solid. m.p. 137-139 ${ }^{\circ} \mathrm{C}$ (EtOAc); FTIR (film/cm ${ }^{-1}$ ): 2934m, 2854m, 1589s, 1566s, 1490s, 1259s, 1179s, 1107s, 990s, 841m, 768m; ${ }^{1} \mathrm{H}$ NMR $\left(300 \mathrm{MHz}, \mathrm{CDCl}_{3}\right): \delta 7.85\left(\mathrm{dd}, J_{1}=7.0 \mathrm{~Hz}, J_{2}=2.0 \mathrm{~Hz}, 2 \mathrm{H}, \mathrm{PhH}\right), 6.91\left(\mathrm{dd}, J_{1}=7.0\right.$ $\left.\mathrm{Hz}, J_{2}=1.9 \mathrm{~Hz}, 2 \mathrm{H}, \mathrm{PhH}\right), 5.98(\mathrm{~s}, 1 \mathrm{H},=\mathrm{CH}), 3.83\left(\mathrm{~s}, 4 \mathrm{H}, \mathrm{NCH}, \mathrm{OCH}_{3}\right), 2.58\left(\mathrm{~s}, 3 \mathrm{H}, \mathrm{SCH}_{3}\right)$, $1.96-1.84(\mathrm{~m}, 6 \mathrm{H}), 1.67(\mathrm{~m}, 1 \mathrm{H}), 1.29-1.24(\mathrm{~m}, 3 \mathrm{H}) \mathrm{ppm} ;{ }^{13} \mathrm{C} \mathrm{NMR}\left(75 \mathrm{MHz}, \mathrm{CDCl}_{3}\right): \delta 172.9$, 166.4, 162.8, 129.1, 125.8, 114.0, 88.6, 61.4, 55.4, 31.1, 26.1, 25.2, 15.3 ppm; LRMS (ESI, positive) $\mathrm{m} / \mathrm{z} 334.1$ [M-19] ${ }^{+}$; Anal.calcd for $\mathrm{C}_{17} \mathrm{H}_{22} \mathrm{BF}_{2} \mathrm{NO}_{2} \mathrm{~S}: \mathrm{C}, 57.80 ; \mathrm{H}, 6.28 ; \mathrm{N}, 3.97$. Found: C, 57.83; H, 6.42; N, 4.02 . 


\section{Acknowledgements}

This work is supported by The National Natural Science Foundation of China (No. 20472086), The National Basic Research Program of China (No. 2009CB526511), The Ministry of Science and Technology and the Ministry of Health of the P.R. China (No. 2009ZX09501-006), and The Chinese Academy of Sciences.

\section{References}

1. (a) Greenhill, J. V. Chem. Soc. Rev. 1977, 6, 277-294. (b) U. Kucklander, U. In The chemistry of enamines; Rappoport, Z. Eds.; New York: Wiley and Sons, 1994; pp 523-636.

2. For reviews on enaminones in organic synthesis, see: (a) Edafiogho, I. O.; Kombian, S. B.; Ananthalakshmi, K. V. V.; Salama, N. N.; Eddington, N. D.; Wilson, T. L.; Alexander, M. S.; Jackson, P. L.; Hanson, C. D.; Scott, K. R. J. Pharm. Sci. 2007, 96, 2509. (b) Ferraz, H. M. C.; Goncalo, E. R. S. Quim. Nova 2007, 30, 957. (c) Al-Zaydi, K. M.; Nhari, L. M. Orient. J. Chem. 2006, 22, 1. (d) Palmieri, G.; Cimarelli, C. Arkivoc 2006, (vi), 104. (e) Svete, J. Arkivoc 2006, (vii), 35. (f) Svete, J. J. Heterocycl. Chem. 2005, 42, 361. (g) Camoutsis, C.; Pairas, G. Trends Heterocycl. Chem. 2003, 9, 237. (h) Aragon, P. J.; Blache, Y. Trends Heterocycl. Chem. 2003, 9, 47. (i) Negri, G.; Kascheres, C.; Kascheres, A. J. J. Heterocycl. Chem. 2004, 41, 461. (j) Svete, J. Monatsh. Chem. 2004, 135, 629. (k) Stanovnik, B.; Steve, J. Chem. Rev. 2004, 104, 2433. (1) Ferraz, H. M. C.; Pereira, F. L. C. Quim. Nova 2004, 27, 89. (m) Kascheres, C. M. J. Braz. Chem. Soc. 2003, 14, 945. (n) Elassar, A.-Z. A.; El-Khair, A. A. Tetrahedron 2003, 59, 8463. (o) Edafiogho, I. O. Saudi Pharm. Journal 2002, 10, 1. (p) Michael, J. P.; De Koning, C. B.; Gravestock, D.; et al. Pure Appl. Chem. 1999, 71, 979. (q) Lue, P.; Greenhill, J. V. Adv. Heterocycl. Chem. 1997, 67, 207. (r) Cimarelli, C.; Palmieri, G. Recent Res. Dev. Org. Chem. 1997, 1, 179. (s) Waldmann, H.; Braun, M. Gazz. Chim. Ital. 1991, 121, 277.

3. For recent development on enaminones in organic synthesis, see: (a) Alnajjar, A.-A.; Abdelkhalik, M. M.; Al-Enezi, A.; Elnagdi, M. H. Molecules, 2009, 14, 68. (b) Al-Saleh, B.; El-Apasery, M. A.; Abdel-Aziz, R. S.; Elnagdi, M. H. J. Heterocycl. Chem. 2005, 42, 563. (c) Al-Mousawi, S.; Abdelkhalik, M. M.; John, E.; Elnagdi, M. H. J. Heterocycl. Chem. 2003, 40, 689. (d) Bartoli, G.; Cupone, G.; Dalpozzo, R.; De Nino, A.; Maiuolo, L.; Procopio, A.; Tagarelli, A. Tetrahedron Lett. 2002, 43, 7441. (e) Katritzky, A. R.; Fang, Y.; Donkor, A.; Xu, J. Synthesis 2000, 2029. (f) Wiberg, K. B.; Snoonian, J. R. J. Org. Chem. 1998, 63, 1390. (g) Bejan, E.; Aït-Haddou, H.; Daran, J.-C.; Balavoine, G. G. A. Eur. J. Org. Chem. 1998, 2907.

4. (a) Edafiogho, I. O.; Kombian, S. B.; Ananthalakshmi, K. V. V.; Salama, N. N.; Eddington, N. D.; Wilson, T. L.; Alexander, M. S.; Jackson, P. L.; Hanson, C. D.; Scott, K. R. J. Pharm. Sci. 2007, 96, 2509. (b) Wilson, T. L.; Jackson, P. L.; Hanson, C. D.; Xue, Z.; Eddington, N. 
D.; Scott, K. R. Med. Chem. 2005, 1, 371. (c) Eddington, N. D.; Scott, K. R. Curr. Top. Med. Chem. 2003, 3, 35. (d) Malawska, B. Curr. Top. Med. Chem. 2005, 5, 69. (e) Salama, N. N.; Eddington, N. D.; Payne, D.; Wilson, T. L.; Scott, K. R. Curr. Med. Chem. 2004, 11, 2093. (f) Eddington, N. D.; Cox, D. S.; Roberts, R. R.; Stables, J. P.; Powell, C. B.; Scott, K. R. Curr. Med. Chem. 2000, 7, 417. (g) Edafiogho, I. O.; Alexander, M. S.; Moore, J. A.; Farrar, V. A.; Scott, K. R. Curr. Med. Chem. 1994, 1, 159.

5. (a) Lee, D. J.; Kim, K. J. Org.Chem. 2004, 69, 4867. (b) Schirok, H.; Alonso-Alija, C.; Michels, M. Synthesis 2005, 3085. (c) Chakrabarti, S.; Panda, K.; Misra, N. C.; Ila, H.; Junjappa, H. Synlett 2005, 1437. (d) Chakrasali, R. T.; Ila, H.; Junjappa, H. Synthesis 1988, 87. (e) Rahman, A.; Vishwakarma, J. N.; Yadav, R. D.; Ila, H.; Junjappa, H. Synthesis 1984, 247. (f) Aggarwal, V.; Singh, G.; Ila, H.; Junjappa, H. Synthesis 1982, 214. (g) Aggarwal, V.; Ila, H.; Junjappa, H. Synthesis 1982, 65. (h) Kumar, A.; Aggarwal, V.; Ila, H.; Junjappa, H. Synthesis 1980, 748.

6. (a) Xia, M.; Wu, B.; Xiang, G. F. J. Fluorine Chem. 2008, 129, 402. (b) Ono, K.; Yoshikawa, K.; Tsuji, Y.; Yamaguchi, H.; Uozumi, R.; Tomura, M.; Taga, K.; Saito, K. Tetrahedron 2007, 63, 9354. (c) Zhang, X.; Yan, C.-J.; Pan, G.-B.; Zhang, R.-Q.; Wan, L.-J. J. Phys. Chem. C 2007, 111, 13851. (d) Maeda, H,; Ito, Y. Inorg. Chem. 2006, 45, 8205. (e) McDonnell, S. O.; O’Shea, D. F. Org. Lett. 2006, 8, 3493. (f) Fujimoto, C.; Kusunose, Y.; Maeda, H. J. Org. Chem. 2006, 71, 2389. (g) Ulrich, G.; Ziessel, R. J. Org. Chem. 2004, 69, 2070. (h) García-Moreno, I.; Costela, A.; Campo, L. J. Phys. Chem. A 2004, 108, 3315. (i) Lai, R. Y.; Bard, A. J. J. Phys. Chem. B 2003, 107, 5036. (j) Ryu, D. H.; Lee, T. W.; Corey, E. J. J. Am. Chem. Soc. 2002, 124, 9992. (k) Vedejs, E.; Chapman, R. W.; Lin, S.; Müller, M,; Powell, D. R. J. Am. Chem. Soc. 2000, 122, 3047. (1) Barluenga, J.; Canteli, R.-M.; Flórez, J.; García-Granda, S.; Gutiérrez-Rodríguez, A.; Martín, E. J. Am. Chem. Soc. 1998, 120, 2514. (m) Lukehart, C. M.; Sacksteder, L. Organometallic, 1987, 6, 150.

7. The crystal structure of $\mathbf{4 f}$ has been deposited at the Cambridge Crystallographic Data Centre and allocated the deposition number: CCDC 756215.

8. Chakrasali, R. T.; Ila, H.; Junjappa, H. Synthesis 1988, 453.

9. $\quad$ Singh, S. J.; Singh, O. M. Tetrahedron Lett. 2008, 49, 3991.

10. Chakrasali, R. T.; Ila, H.; Junjappa, H. Synthesis 1988, 851.

11. Kohra, S.; Turuya, S.; Kimura, M.; Ogata, K.; Tominaga, Y. Chem. Pharm. Bull. 1993, 41, 1293.

12. Gupta, A. K.; Chakrasali, R. T.; Ila, H.; Junjappa, H. Synthesis 1989, 141.

13. Pooranchand, D.; Ila, H.; Junjappa, H. Synthesis 1987, 547. 